\title{
Trichoplax adhaerens reveals a network of nuclear receptors sensitive to 9-cis-retinoic acid at the base of metazoan evolution
}

\author{
Jan Philipp Novotný ${ }^{1,2}$ ， Ahmed Ali Chughtai ${ }^{1}$ ， Markéta Kostrouchová ${ }^{1,3}$ ， Veronika Kostrouchová ${ }^{1}$, David \\ Kostrouch ${ }^{1}$ ， Filip Kaššák ${ }^{4}$, Radek Kaňa ${ }^{5}$, Bernd Schierwater ${ }^{6,7}$, Marta Kostrouchová ${ }^{1}$, Zdenek Kostrouch \\ Corresp. 1 \\ 1 Biocev, First Faculty of Medicine, Charles University, Vestec, Czech Republic \\ 3 Department of Pathology, Third Faculty of Medicine, Charles University, Prague, Czech Republic \\ 4 Biocev, Charles University, Vestec, Czech Republic \\ 5 Institute of Microbiology, Laboratory of Photosynthesis, Czech Academy of Sciences, Třeboň, Czech Republic \\ 6 Institute for Animal Ecology and Cell Biology, University of Veterinary Medicine, Hannover, Germany \\ 7 Department of Molecular, Cellular and Developmetnal Biology, Yale University, New Haven, CT, United States \\ Corresponding Author: Zdenek Kostrouch \\ Email address: Zdenek.kostrouch@If1.cuni.cz
}

Trichoplax adhaerens, the only known species of Placozoa is likely to be closely related to an early metazoan that preceded branching of Cnidaria and Bilateria. This animal species is surprisingly well adapted to free life in the World Ocean inhabiting tidal costal zones of oceans and seas with warm to moderate temperatures and shallow waters. The genome of T. adhaerens (sp. Grell) includes four nuclear receptors, namely orthologue of RXR (NR2B), HNF4 (NR2A), COUP-TF (NR2F) and ERR (NR3B) that show a high degree of similarity with human orthologues. In the case of RXR, the sequence identity to human RXR alpha reaches $81 \%$ in the DNA binding domain and $70 \%$ in the ligand binding domain. We show that $T$. adhaerens RXR (TaRXR) binds 9-cis retinoic acid (9-cis-RA) with high affinity, as well as high specificity and that exposure of $T$. adhaerens to 9-cis-RA regulates the expression of the putative $T$. adhaerens orthologue of vertebrate L-malate-NADP ${ }^{+}$oxidoreductase (EC 1.1.1.40) which in vertebrates is regulated by a heterodimer of RXR and thyroid hormone receptor. Treatment by 9-cis-RA alters the relative expression profile of $T$. adhaerens nuclear receptors, suggesting the existence of natural ligands. Keeping with this, algal food composition has a profound effect on $T$. adhaerens growth and appearance. We show that nanomolar concentrations of 9-cis-RA interfere with $T$. adhaerens growth response to specific algal food and causes growth arrest. Our results uncover an endocrine-like network of nuclear receptors sensitive to 9-cis-RA in T. adhaerens and support the existence of a ligand-sensitive network of nuclear receptors at the base of metazoan evolution. 
1 Trichoplax adhaerens reveals a network of nuclear receptors sensitive

2 to 9-cis-retinoic acid at the base of metazoan evolution.

3

4 Jan Philipp Novotný ${ }^{1,2}$, Ahmed Ali Chughtai ${ }^{1}$, Markéta Kostrouchová ${ }^{1,3}$, Veronika Kostrouchová ${ }^{1}$,

5 David Kostrouch ${ }^{1}$, Filip Kašš́́k ${ }^{1}$, Radek Kaňa ${ }^{4}$, Bernd Schierwater ${ }^{5,6}$, Marta Kostrouchová ${ }^{1}$, 6 Zdeněk Kostrouch ${ }^{1 *}$

7

8

91 Biocev, First Faculty of Medicine, Charles University, Vestec, Czech Republic

102 Department of Medicine V, University of Heidelberg, Heidelberg, Germany

113 Department of Pathology, Third Faculty of Medicine, Charles University, Ruská 87, 10000 Praha

12 10, Czech Republic

134 Laboratory of Photosynthesis, Centrum Algatech, Institute of Microbiology, Academy of

14 Sciences Novohradska 237 - Opatovicky mlýn, CZ 37901 Třeboň, Czech Republic.

155 Division of Ecology and Evolution, Stiftung Tierärztliche Hochschule, Hannover Germany

166 Department of Molecular, Cellular and Developmental Biology, Yale University

17 New Haven, CT, USA

$19 *$ Corresponding author: 
20

21

22

23

24

25

26

27

28

29

30

31

32

33

34 35 36
Zdenek Kostrouch

Biocev, First Faculty of Medicine,

Charles University,

Průmyslová 595, 25242 Vestec,

Czech Republic

email: zdenek.kostrouch@,lf1.cuni.cz

Trichoplax adhaerens, the only known species of Placozoa is likely to be closely related to an early metazoan that preceded branching of Cnidaria and Bilateria. This animal species is surprisingly well adapted to free life in the World Ocean inhabiting tidal costal zones of oceans and seas with warm to moderate temperatures and shallow waters. The genome of T. adhaerens (sp. Grell) includes four nuclear receptors, namely orthologue of RXR (NR2B), HNF4 (NR2A), COUP-TF (NR2F) and ERR (NR3B) that show a high degree of similarity with human orthologues. In the case of RXR, the sequence identity to human RXR alpha reaches $81 \%$ in the DNA binding domain and $70 \%$ in the ligand binding domain. We show that T. adhaerens RXR (TaRXR) binds 9-cis retinoic acid (9-cis-RA) with high affinity, as well as high specificity and that exposure of T. adhaerens to 9-cis-RA regulates the expression of the putative $T$. adhaerens orthologue of vertebrate L-malate-NADP ${ }^{+}$oxidoreductase (EC 1.1.1.40) which in vertebrates is regulated by a heterodimer of RXR and thyroid hormone receptor. Treatment by 9-cis-RA alters 
43 the relative expression profile of $T$. adhaerens nuclear receptors, suggesting the existence of

44 natural ligands. Keeping with this, algal food composition has a profound effect on T. adhaerens

45 growth and appearance. We show that nanomolar concentrations of 9-cis-RA interfere with $T$.

46 adhaerens growth response to specific algal food and causes growth arrest. Our results uncover an

47 endocrine-like network of nuclear receptors sensitive to 9-cis-RA in T. adhaerens and support the

48 existence of a ligand-sensitive network of nuclear receptors at the base of metazoan evolution.

\section{INTRODUCTION}

52

53 Life on Earth began 4.1 to 3.5 billion years ago (Bell et al. 2015) with the appearance of the first

54 unicellular organisms that subsequently evolved, in part, to multicellular lifeforms forming the

55 kingdom Metazoa that have specialized tissues for digestion, regulation of homeostasis,

56 locomotion, perception, analysis of the environment and reproduction.

57 In contrast to unicellular organisms, metazoans are in need of regulatory mechanisms that

58 provide the means of coordination between various tissues in a tight arrangement with cellular

59 homeostasis. This coordination on the level of humoral signaling includes regulation by nuclear receptors (NRs), which respond to small, mostly hydrophobic molecules, including hormones produced by specific tissues, metabolites or even molecules present in the environment and transfer these signals to the nucleus, and thus leading to a dynamically changing but adaptive gene expression (Escriva et al. 2004).

NRs therefore play an important role in maintaining intra- and inter-cellular functions in 
66 of an A/B (N-terminal) domain, the DNA binding domain (DBD), a hinge region, the ligand

67 binding domain (LBD) and the C-terminal domain (Kumar \& Thompson 1999; Robinson-Rechavi

68 et al. 2003). The DBD and LBD of NRs exhibit an especially high degree of conservation and the

69 changes that were acquired during evolution allow classification of the NR protein family into six

70 subfamilies (Laudet 1997; Escriva et al. 1998). NRs that evolved within these subfamilies show

71 functional connections that include specialization of regulatory functions in time or cell type

72 restriction, fortification of ancestral functions or their specific inhibition by newly evolved NRs

73 (Escriva et al. 2004; Kostrouchova \& Kostrouch 2015).

74 With the overall structure maintained across metazoan species, nuclear receptors show 75 significant heterogeneity regarding their quantity and function, many of which have not yet been 76 explored in e.g. Caenorhabditis elegans with over 280 nuclear receptors (reviewed in 77 (Kostrouchova \& Kostrouch 2015)).

The evolutionary changes accumulated in diversified NRs allow functional

79 subspecialization at the level of specific sequence binding within gene promoters (response elements), protein-protein interactions with functionally linked receptor interactors and adoption

81 of new ligands as specific hormonal regulators. The evolution of hormonal ligands acquired by

82 different species during evolution is well documented and indicates the potential of NRs to adopt

83 new ligands as regulators (Escriva et al. 2000; Markov \& Laudet 2011). Indeed, it is now thought

84 that NRs evolved as environmental sensors that were able to sense a wide variety of compounds 85 with low affinity and specificity, some of which later-on acquired higher affinity binding towards 86 certain ligands that are products of metabolic pathways (Holzer et al. 2017). This can be 87 exemplified by the high affinity and specificity binding of certain receptors, such as the 88 mineralocorticoid or androgen receptors, while the family of PPARs shows a rather promiscuous 
89 binding to a variety of different substances (Issemann \& Green 1990). Keeping in mind the 90 metabolic origin of NR ligands, it is not completely surprising to see different ligands binding to 91 orthologues across species, such as Triac and T3 in the case of TR (Escriva 2008, Bridgham 2008), 92 thus changing in the course of evolution and adapting to new environments.

This is accompanied by the essential questions, to what degree the plasticity of ligand

94 selection is a fundamental property of NRs and what the origin of specific ligand binding by NRs might be. It has been suggested that the original NR, which is the ancestral NR possessing gene regulatory capacity, may have been an unliganded molecular regulator (Escriva et al. 1997). However, it is now believed that the ancestral NR is most closely related to the NR2 subfamily, as members of this family can be found in basal metazoans and are sensors of fatty acids (Holzer et al. 2017). More recently, it was proposed that the ligand binding and ligand-dependent regulatory potential of NRs is an inherent feature of the evolution of NRs (Bridgham et al. 2010). Due to their nature of fine tuning cellular responses in response to environmental changes without necessarily showing high affinity binding to a set of ligands, cross-species comparison of nuclear receptor networks might shed light on the details of the NR network function (Holzer et al. 2017). to the discovery of an RXR orthologue in Cnidaria (Kostrouch et al. 1998). Surprisingly, this receptor showed not only extremely high degree of sequence identity with vertebrate RXRs, far surpassing the degree of conservation observed in insects but also by its ability to bind the same ligand as vertebrate RXRs, 9-cis-retinoic acid (9-cis-RA), with an affinity close to that reported

109 for vertebrate RXRs. Similarly, as vertebrate RXRs, the jellyfish RXR showed a specific binding 110 preference for 9-cis-RA over all-trans-retinoic acid (AT-RA) and was able to heterodimerize with 111 vertebrate thyroid hormone receptor alpha. Recent genome sequencing projects confirmed the 
112 existence of highly conserved RXR across several metazoan species including insects (Locusta

113 migratoria (Nowickyj et al. 2008), that are evolutionarily older than species with a more

114 diversified RXR orthologue such as Usp found in Drosophila (reviewed in (Gutierrez-Mazariegos

115 et al. 2014)).

116 To date, the nuclear receptor network has mainly been studied in complex organisms

117 already in possession of an extensive endocrine network. Albeit dissection of nuclear receptor

118 networks in these organisms can outline functions and associated regulatory cascades, basal tasks

119 might be obscured by the gain of further, more specialized functions. Genome analysis of the basal

120 metazoan Trichoplax adhaerens by whole genome sequencing revealed four highly conserved

121 nuclear receptors, namely orthologues of HNF4 (NR2A), RXR (NR2B), ERR (NR3B) and COUP-

122 TF (NR2F) (Baker 2008; Srivastava et al. 2008) and thus allows assessment of the most basal

123 workings of nuclear receptors. Surprisingly, the degree of conservation of the predicted placozoan

124 NRs with known vertebrate NRs is not only very high at the level of the predicted secondary

125 structure, as can be expected for true NRs, but also at the level of the primary amino acid sequence.

126 Especially the similarity of the placozoan RXR (TaRXR) to its vertebrate orthologues is high, as

127 it is in the case of the cubomedusan RXR. T. adhaerens, which shows characteristics of a basal

128 metazoan with only a few cell types (Smith et al. 2014) and a relatively simple 4 member NR

129 complement, offers a unique model that may shed light on the evolution of gene regulation by 130 NRs.

131 In this presented work, we attempted to study the placozoan RXR orthologue functionally.

132 Our results show that $T$. adhaerens RXR binds 9-cis-RA with an affinity comparable to that of

133 vertebrate and jellyfish RXRs and that $T$. adhaerens responds to nanomolar concentrations of 9-

134 cis-RA with a transcriptional upregulation of the putative orthologue of a malic enzyme that is 
135 regulated by a heterodimer formed by liganded thyroid hormone receptor and RXR in vertebrates.

136 We also show that 9-cis-RA affects the relative expression of the four NRs present in T. adhaerens

137 genome suggesting that these NRs may form a regulatory network capable of responding to

138 possible ligands present in these animals or their environment. In line with this, growth,

139 multiplication and appearance of $T$. adhaerens are strongly affected by food composition,

140 especially by red pigment containing algae suggesting that specific food components or their

141 metabolites may be ligands involved in the ancestral regulatory network of NRs. In support of this,

$1423.3 \mathrm{nM}$ 9-cis-RA interferes with T. adhaerens growth response to the feeding by Porphyridium

143 cruentum and causes balloon-like phenotypes and death of animals while animals fed by Chlorella

$144 s p$. are partially protected against the treatment by $3.3 \mathrm{nM} 9-c i s-\mathrm{RA}$, do not develop balloon-like

145 phenotypes but are also arrested in their growth and propagation indicating that 9-cis-RA interferes

146 with $T$. adhaerens growth and development.

147

148

149 METHODS

150 Bioinformatics and cloning of RXR

The predicted RXR gene models on jgi (http://jgi.doe.gov/) (Nordberg et al. 2014) were

152 screened for the characteristic molecular signature of the DNA binding domain (C-X2-C-X13-C-

153 X2-C-X15-C-X5-C-X9-C-X2-C-X4-C-X4-M) (Kostrouch et al. 1995) and the appropriate

154 predicted gene model (protein ID 53515) was selected for further use.

155

The alignment of different RXRs was performed by Clustal Omega

156 (http://www.ebi.ac.uk/Tools/msa/clustalo/) (Sievers et al. 2011) and adjusted/exported as an

157 image file using Jalview (http://www.jalview.org). Protein domain characterization was performed 
158 with SMART (Schultz et al. 1998; Letunic et al. 2015). Analysis of HNF4, ERR and COUP-TF

159 was done similarly. Phylogenetic analysis was performed on RXR ClustalO alignment using

160 PhyMLv3.1 (Guindon et al. 2010) implemented in SeaView v4.6.1 with a 100 bootstrap analysis

161 and SPR distance computation. The tree was then visualized using FigTree v1.4.3.

162

T. adhaerens total RNA was obtained from 50-100 pooled individual animals and extracted

163 using TRIZOL $®$ reagent (Invitrogen, Carlsbad, CA, USA) according to the protocol supplied by

164 the manufacturer.

165 Subsequently, cDNA was prepared with random hexamers and SuperScript III 166 (Invitrogen ${ }^{\mathrm{TM}}$ ) according to the manufacturer's protocol.

167 Several RXR transcripts were then amplified by PCR with primers covering the starting sequence 168 ((GCGGATCC)ATGGAGGACAGATCGTTTAAAAAA), starting at 32 bp 5' of ATG 169 (TCTACCAATGTTTATCGCATCGGTTA) and starting at 97 bp 5, of ATG 170 (TTAAGGCTTAACTGATGATGTTGTGAATG) with a common reverse primer covering the 171 last 24 bp of the predicted gene sequence

172 ((CGGAATTC)TTAAGAACTGCCTGTTTCCAGCAT).

173 Each PCR product was then ligated into pCR $\AA 2.1-\mathrm{TOPO} \AA$ or $\mathrm{pCR} \AA 4-\mathrm{TOPO} \AA$ vector 174 with the classic TA Cloning Kit and TOPO TA Cloning Kit (Invitrogen ${ }^{\mathrm{TM}}$ ), respectively. The 175 ligated products were then transformed using One Shot ${ }^{\circledR}$ TOP10 Chemically Competent E. coli 176 and cultured on LB Agar plates containing $100 \mu \mathrm{g} / \mathrm{ml}$ ampicillin. Plasmid DNA was extracted 177 from obtained colonies and screened for mutations by sequencing using vector specific M13 178 forward and reverse primers. Only non-mutated sequences were used in subsequent experiments.

179 The RXR fragments were then restricted and inserted into pGEX-2T vector system for bacterial 180 expression (Addgene, Cambridge, MA, USA). Proper insertion was verified by sequencing. 


\section{Protein expression}

183

B121 pLysS bacteria were transformed with previously described RXR mRNA inserted

184 into pGEX-2T vector. Stocks of transformed bacteria were stored in $8 \%$ glycerol according to the

185 Novagen $\quad$ PET $\quad$ System $\quad$ Manual $11^{\text {th }}$ edition)

186 (https://www.google.cz/search?q=Novagen + pET + System + Manual $+\& i e=u t f-8 \& o e=u t f-$

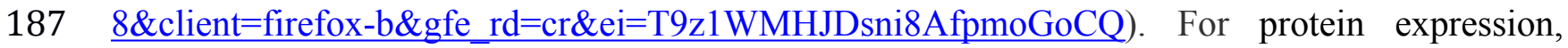

188 bacteria were scraped from stock and incubated in Liquid Broth (LB) with ampicillin (100 $\mu \mathrm{g} / \mathrm{ml})$

189 and chloramphenicol $(34 \mu \mathrm{g} / \mathrm{ml})$ overnight. The culture was then used to inoculate $100 \mathrm{ml}$ of LB

$190+$ antibiotics and grown to $\mathrm{OD} 600=0.6-0.8$ at $37^{\circ} \mathrm{C}$, then induced with $100 \mu 1 \mathrm{M}$ IPTG

191 (isopropyl-D-thiogalactopyranoside) (Sigma-Aldrich, St. Louis, MO, USA) and moved to $25^{\circ} \mathrm{C}$

192 (RT) for $5 \mathrm{~h}$. The culture was then spun at $9000 \mathrm{xg}$ for $15 \mathrm{~min}$ and the supernatant discarded. The

193 bacterial pellet was resuspended in $10 \mathrm{ml} \mathrm{GST}$ binding buffer $(25 \mathrm{mM}$ Tris pH 7.5, $150 \mathrm{mM} \mathrm{NaCl}$,

$1941 \mathrm{mM}$ EDTA + protease inhibitor (S8820 Sigma Fast, Sigma-Aldrich, St. Louis, MO, USA or

195 cOmplete ${ }^{\mathrm{TM}}$, EDTA-free Protease Inhibitor Cocktail, Roche, Basel, Switzerland). Bacteria were

196 then lysed by 6 × 20 s ultrasonication on ice ( 50 watts, $30 \mathrm{kHz}$, highest setting - 100\%) (Ultrasonic

197 Processor UP50H, (Hielscher Ultrasonics GmbH, Teltow, Germany) and subsequently incubated

198 with 15-20 mg glutathione agarose beads (Sigma-Aldrich $\left.{ }^{\circledR}\right)$ prepared according to manufacturer's

199 instructions. Incubation took place at $4{ }^{\circ} \mathrm{C}$ for about $10 \mathrm{~h}$ after which the beads were washed

200 according to instructions, resuspended in regeneration buffer $(50 \mathrm{mM}$ Tris- $\mathrm{HCl} \mathrm{pH} 7.4,1 \mathrm{mM}$

201 EDTA, $120 \mathrm{mM} \mathrm{KCl,} 5 \mathrm{mM}$ DTT, 8\% glycerol (v/v)) or 50mM TRIS-HCl pH $7.4+9 \%$ (v/v)

202 glycerol for subsequent thrombin (bovine plasma, Sigma-Aldrich ${ }^{\circledR}$ ) cleavage, if performed, and

203 then adjusted for regeneration buffer conditions. GST-TaRXR was eluted from glutathione agarose 
204 beads using $10 \mathrm{mM}$ reduced glutathione (Sigma-Aldrich, StLouis, Mo, USA) in $50 \mathrm{mM}$ Tris-HCl

205 buffer $\mathrm{pH}$ 8.0. The size of the GST-TaRXR fusion protein was checked by polyacrylamide gel

206 electrophoresis. Thrombin cleavage was performed at RT for $4 \mathrm{~h}$ and the quality of the purified

207 protein was assessed by polyacrylamide gel electrophoresis.

208

209

210

211

212

213

214

215

216

217

218

219

220

221

222

223

224 indirect illumination with a 60W, 120V yellow light bulb (BugLite, General Electric Co, Nela

225 Parc, Cleveland Oh, USA) as described (Cahnmann 1995). The radioactivity of the GST-fusion

226 protein and cleaved protein was measured using Packard Tri-Carb 1600TR Liquid Scintillation 
227 Analyzer (Packard, A Canberra Company, Canberra Industries, Meriden, CT, USA) and Ultima

228 Gold Scintillation Fluid (PerkinElmer, Waltham, MA, USA). The fraction of bound ${ }^{3} \mathrm{H}-$ labelled

229 9-cis-RA and ${ }^{3} \mathrm{H}$-labelled all-trans-RA was determined as a ratio of the bound radioactivity of 230 precipitated GST-TaRXR / total radioactivity used at the particular condition (determined as the 231 sum of bound radioactivity and the total radioactivity of collected wash fluids) in the absence of 232 non-radioactive competitors or 200 fold excess of 9-cis-RA and all-trans-RA in the case of ${ }^{3} \mathrm{H}-$ 233 labelled 9-cis-RA and 40 fold excess of non-radioactive competitors in the case of ${ }^{3} \mathrm{H}-$ labelled all234 trans-RA (to compensate for the higher affinity of 9-cis-RA compared to all-trans-RA in binding 235 to TaRXR ).

236

\section{Culture of $\boldsymbol{T}$. adhaerens and algae}

238 Trichoplax adhaerens was cultured in Petri dishes containing filtered artificial seawater 239 (Instant Ocean, Spectrum Brands, Blacksburg, VA, USA) with a salinity of approx. 38-40 ppt. 240 Rhodomonas salina (strain CCAP 978/27), Chlorella sp., Porphyridium cruentum (UTEX B637)

241 and other non-classified algae, as well as aquarium milieu established in the laboratory by mixing 242 salt water obtained from a local aquarium shop were used to maintain the stock. The cultures were 243 kept at approx. $23^{\circ} \mathrm{C}$ and an automated illumination for $12 \mathrm{~h} /$ day was used with a conventional 244 light bulb on a daylight background from late spring to mid-summer in the laboratory located at $24550.07031 \mathrm{~N}, 14.42934 \mathrm{E}$ with laboratory windows oriented eastward. The natural illumination 246 included almost direct morning light from $8 \mathrm{AM}$ to $10.30 \mathrm{AM}$, indirect sunlight for most of the 247 daytime and sunlight reflected from a building across the street from 1 PM to 6 PM. Algae were 248 maintained as described (Kana et al. 2012; Kana et al. 2014). The experiments were performed 249 predominantly during sunny weather. 


\section{Treatment of $\boldsymbol{T}$. adhaerens with retinoic acids}

Incubation of the animals was done overnight in the absence of light. Each batch within an

253

254

255

256

257

258

259

260

261

262

263

264

265

266

267

268

269

270

271

\section{Quantitative PCR} sp. at 72,90 and $378 \mathrm{~h}$.

experiment was derived from similar cultures and fed with similar amounts and composition of algae. All experiments were started in a dark room with indirect yellow light illumination (similarly as in the case of the ligand binding studies) and further incubations were done in the dark for 24 hours. In experiments aimed at the visualization of 9-cis-RA effect on T. adhaerens response to feeding conditions, parallel cultures were set and fed with $P$. cruentum. Large animals of approximately the same size were individually transferred to new control and experimental cultures and fed with $P$. cruentum algal cells. After 6 hours of incubation under natural indirect illumination, all animals in both control and experimental cultures were photographed (max. magnification on Olympus SZX7 with Olympus E-410 camera) and the final volume of cultures was adjusted to $50 \mathrm{ml}$ (determined by the weight of cultures in $110 \mathrm{~mm}$ glass Petri dishes). Next, the room was darkened and further manipulations were done under indirect illumination with yellow light. Five $\mu 1$ of vehicle (1\% DMSO in ethanol) or vehicle containing 9-cis-RA was added into $50 \mathrm{ml}$ of final volume to the final concentration of 9-cis-RA $3.3 \mathrm{nM}$. Similarly, parallel subcultures were prepared from slowly growing cultures fed by microorganisms covering glass slides in an equilibrated 251 laboratory aquarium and fed by Chlorella sp. Cultures were incubated in the dark for $24 \mathrm{~h}$ and all animals were counted under microscope and photographed again. The cultures were then left under natural illumination and cultured for an additional two or three weeks. Animals fed by P. cruentum were measured again at 72, 90 and $450 \mathrm{~h}$ and those fed by Chlorella 
273 Droplet digital PCR was performed on a QX100 Droplet Digital PCR System (Bio-Rad

274 Laboratories, Hercules, CA, USA). For this, T. adhaerens was cultured according to culture

275 conditions described and 4-10 animals removed per 100 $\mu 1$ TRIZol reagent (Invitrogen, Carlsbad,

276 CA, USA). Total RNA was measured by a UV spectrophotometer and used as a reference for 277 normalization.

278 Reverse transcription was performed with SuperScript III Reverse Transcriptase 279 (ThermoFisher, Waltham, MA, USA) according to manufacturer's instructions. The cDNA 280 (corresponding to 100-500 ng of RNA) was then mixed with ddPCR Supermix (Bio-Rad, Hercules, 281 CA, USA) according to the manufacturer's instructions and analyzed. PCR primers were designed 282 using the UPL online ProbeFinder (Roche) software and were as follows:

283 TaRXR - left:tctgcaagttggtatgaagca, right: agttggtgtgctattctttacgc

284 TaHNF4 ( ref|XM_002115774.1|):

285 left: ggaatgatttgattttacctcgac, right: tacgacaagcgatacgagca

286 TaCOUP-TF ( $\left.\underline{\text { ref } \mid X M \_002109770.1}\right):$

287 left: attttgaatgctgcccaatg, right: ttactggttgtggagtatggaaac

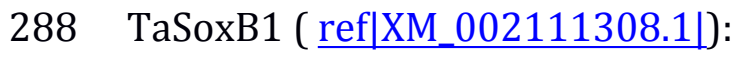

289 left: tgtcagatgcggataaacga, right: ggatgttccttcatgtgtaatgc

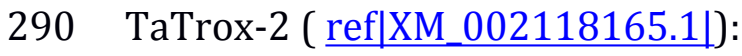

291 left: gcctatagtcgatcctgccata, right: ttggtgatgatggttgtcca

292 TaPaxB1 ( gb|DQ022561.1):

293 left: tcaaacgggttctgttagcc, right: ggtgttgccaccttaggc

294 TaERR (nuclear receptor 3, gb|KC261632.1):

295 left: ttacgcatgtgatatggttatgg, right: agcgtgcctatttatttcgtct 
Results were subsequently analyzed using the Bio-Rad ddPCR software. Manual

298

299

300

301

302

303

304

305

306

307

308

309

310

311

314

315

\section{Microscopy and image analysis} expressional analysis. experiment.

correction of the cut off was performed when automated analysis was not possible. To visualize changes in nuclear receptor expression in the absence of a reliable housekeeping gene as a reference, we considered the absolute quantity of each nuclear receptor as a percentage of the overall nuclear receptor expression and subsequently visualized the change of receptor expression by subtraction of the percentage of the control experiment. Absolute copy numbers of the proposed malic enzyme orthologue in T. adhaerens have been normalized to overall RNA quantity for

Experiments with quantification by qRT-PCR were performed on a Roche LightCycler II with OneTaq polymerase and the same probes as for ddPCR.

For the estimation of the relative expression of NRs in small $(<0.5 \mathrm{~mm})$ versus big animals (>1 mm), 20 to 30 animals from the same culture were used for each paired

\section{Identification of $T$. adhaerens orthologue of L-malate-NADP ${ }^{+}$oxidoreductase (EC 1.1.1.40)}

P48163 (MAOX_HUMAN) protein sequence was used as the query sequence and searched against T. adhaerens database with BLASTP on http://blast.ncbi.nlm.nih.gov/Blast.cgi using standard algorithm parameters. The best hit was a hypothetical protein TRIADDRAFT_50795 with a sequence identity of 57\% and a query coverage of $93 \%$ and was assumed to be $T$. adhaerens closest orthologue of vertebrate L-malate-NADP ${ }^{+}$oxidoreductase. 
320 DF Plan 2x objective and Olympus DP 73 camera operated by CellSens Dimension computer

321 program (kindly provided by Olympus, Prague, Czech Republic) or Olympus CKX41 or SZX7

322 with Olympus E-410 camera and QuickPhoto Micro 3.1 program.

323 Circularity was calculated by establishing the area (A) and perimeter (p) of T. adhaerens

324 using ImageJ (https://imagej.nih.gov/ij/) and then calculated with the isoperimetric quotient $Q=$

$325 \frac{4 \pi A}{p^{2}},(\mathrm{~A}-\mathrm{Area}, \mathrm{p}-$ perimeter). GraphPad Prism 5 (or higher) was used for graphical representation

326 and calculations of the confidence intervals with $\mathrm{p}=0.05$.

\section{RESULTS}

\section{T. adhaerens retinoid $X$ receptor shows high cross-species sequence identity}

By using the ab initio model of the JGI Trichoplax database as a reference, we screened

333 the Trichoplax JGI database for RXR orthologues with a complete DBD and LBD sequence and

334 were able to obtain, as well as verify a full length RXR transcript previously not annotated as the

335 'best model'. Blastp analysis showed a high sequence similarity to human, as well as mouse RXR 336 with $66 \%$ overall sequence identity to human RXR alpha.

SMART analysis of the proposed TaRXR sequence showed a zinc finger DNA binding domain (amino acid residues 16-87) and a ligand binding domain (amino acid residues 155-342) with E values $<10^{-40}$. Blast analysis of the zinc finger DNA binding and ligand binding domains revealed a sequence identity of $81 \%$ and $70 \%$ to human RXR alpha, respectively. Both domains

341 contained the predicted molecular pattern characteristic for each domain. The heptad repeat 
342 LLLRLPAL proposed for dimerization activity (Forman \& Samuels 1990b; Forman \& Samuels

343 1990a; Kiefer 2006) as well as the LBD signature for 9-cis-RA binding Q-x(33)-L-x(3)-F-x(2)-R-

344 x(9)-L-x(44)-R-x(63)-H (Egea et al. 2000) were present (Fig. 1). From 11 amino acid residues

345 shown to be critical for 9-cis-RA binding (A271, A272, Q 275, L 309, F 313, R 316, L 326, A 327,

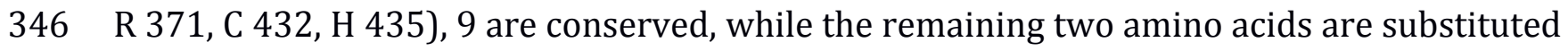

347 [A327S, and C432T (C432A in Tripedalia cystophora)]. Due to the high sequence identity, we

348 propose a 9-cis-retinoic acid binding capability of the hypothesized TaRXR sequence, as well as

349 DNA binding capability. Phylogenetic analysis using PhyML algorithm indicates that TaRXR is

350 likely to precede branching of RXRs in cubomedusae and scyphomedusae and clusters with RXRs

351 in bilateria (Supplementary Fig S1).

352 The remaining three NRs identified in the T. adhaerens genome show also very high

353 overall sequence identity with vertebrate orthologues. Alignments of T. adhaerens HNF4, COUP-

354 TF and ERR with orthologues from selected species can be found in Supplementary File S2.

355

356

357

TaRXR shows preferential binding affinity to 9-cis retinoic acid over all-transretinoic acid

In order to analyze the binding properties of TaRXR, we expressed TaRXR as a GSTfusion protein (GST-TaRXR) in bacteria which was then purified as a GST-fusion protein and used directly for binding studies or cleaved by thrombin and eluted as TaRXR. The binding of ${ }^{3} \mathrm{H}-$ labelled 9-cis-RA or ${ }^{3} \mathrm{H}$-labelled all-trans-RA was determined by measuring total bound radioactivity and the radioactivity displaceable by 200 fold excess of nonradioactive competitors.

364 Consistent with the high conservation of the LBD, the experiments showed that TaRXR prepared 
365 as thrombin cleaved TaRXR or GST-TaRXR binds 9-cis-RA with high affinity and specificity

366 (Fig. 2 A and B). The 9-cis-RA binding assay showed high affinity binding to GST-TaRXR with

367 a saturation plateau from $5 \mathrm{nM}$ to $10 \mathrm{nM}$ (Fig. 2 C). In contrast, all-trans-retinoic acid did not

368 show high affinity binding to TaRXR of GST-TaRXR.

369

370

9-cis-retinoic acid induces malic enzyme gene expression at nanomolar

371

372

373

374

375

376

377

378

379

380

381

382

383

384

385

386 387

\section{concentrations}

Next, we searched whether 9-cis-RA has observable biological effects on T. adharens at nanomolar concentrations. We hypothesized that TaRXR is likely to be involved in the regulation of metabolic events. In vertebrates, RXR is a dimerization partner of TR and together, these two NRs are regulating a wide range of metabolic pathways. We, therefore, searched for an orthologue of vertebrate L-malate-NADP ${ }^{+}$oxidoreductase (EC 1.1.1.40) in T. adhaerens genome since this enzyme is an established reporter of the state of thyroid hormone dependent regulation (see Discussion).

The sequence of the $T$. adhaerens likely orthologue of vertebrate L-malate-NADP ${ }^{+}$ oxidoreductase was retrieved from the Trichoplax genomic database together with its presumed promoter based on the predicted sequence (Supplementary File S3).

Droplet digital PCR showed an increased transcription of the predicted L-malate-NADP ${ }^{+}$ oxidoreductase gene after incubation of T. adhaerens with 9-cis-RA, but not with all-trans-RA (Fig. 3). In repeated experiments, we observed that the level of induction was higher at 9-cis-RA concentrations in the range of 1 to $10 \mathrm{nM}$, than above $10 \mathrm{nM}$. We also noticed that the level of the induction slightly varied based on the actual $T$. adhaerens cultures and the algal food composition of the T. adhaerens cultures. 
Changes in the culture environment alter the expression pattern of the nuclear

391

392

393

394 395

396

397

398

399

400

401

402

403

404

405

406

407

408

409

410

\section{receptor complement in $T$. adhaerens}

From the experience we gained by culturing $T$. adhaerens, as well as from the previous experiments we knew that the culture conditions could dramatically influence phenotype. Having the possible developmental functions of the ancestral NRs in mind, we raised the question whether the expression patterns of the NRs reflect changes in phenotype.

Firstly, we assayed the relative expression of RXR against all three other NRs in small versus big animals $(<0.5 \mathrm{~mm}$ or $>1 \mathrm{~mm})$. The relative proportion of the RXR expression compared to the remaining NRs was found to be higher in big animals (33\%) than in small animals (24\%). The treatment by $3.3 \mathrm{nM}$ 9-cis-RA led to a dramatic increase of the relative expression of RXR in comparison to the rest of the NR complement (51\%), indicating that phenotypic changes are connected with differential expression of NRs and that 9-cis-RA affects the expression of RXR.

In order to see the effect of 9-cis-RA on all NRs, we sampled and extracted RNA from cultures containing the same number of big and small animals treated with different concentrations of 9-cis-RA. The experimental cultures were started from the same original cultures and during incubation were fed with Chlorella sp. only since this algal food showed to have the least effect on T. adhaerens cultures. All four T. adhaerens NRs were quantified by either qRT-PCR or ddPCR.

Analysis of NR expression pattern in animals incubated with different concentrations of 9cis-RA, revealed a relative increase in RXR expression at low nanomolar concentrations $(<10 \mathrm{nM})$ in repeated experiments. In contrast, further increase of 9-cis-RA resulted in smaller changes 
411 compared to the expression pattern of NRs in control animals or even reverted the values observed

412 in low nanomolar conditions (Fig. 4).

413

414

415 Food composition dramatically changes the phenotype and the reproduction

416 rate of $\boldsymbol{T}$. adhaerens

T. adhaerens retrieved from laboratory aquariums used for the stock cultures were

relatively similar in appearance and included small round animals containing approximately 50

cells and grew to animals with an approximate diameter of $0.2 \mathrm{~mm}$ and rarely were bigger. Their

420

rate of multiplication when transferred to Petri dishes was doubling in one month or even one

421

week, depending on whether the glass was covered by microbial and algal films established during

422 culturing in aquariums. We attempted to use several defined algae as artificial food. They included

423 Pyrrenomonas helgolandii, Picocystis salinarium, Tetraselmis subcoriformis, Rhodomonas

424 salina, Phaeodactylum tricornutum, Porphyridium cruentum and Chlorella sp. Individual

425 subcultures of $T$. adhaerens differed in the rate of propagation and appearance as well as colors

426 that were varying from greenish to brown and reddish taints depending on the food that was used

427 as singular species food or mixtures (Fig. 5). Also, contaminants from the original algal food,

428 which prevailed in some cultures, had an influence on T. adhaerens growth and behavior. In

429 controlled experiments, it became clear that some food components or their metabolites are

430 influencing growth and appearance of T. adherens more than food availability. When T. adhaerens

431 were fed with equal amounts of algal cells (although they differed in size and expected

432 digestibility), the addition of algae containing red pigments - Cryptophytes (Pyrrenomonas

433 helgolandii and Rhodomonas salina) or Rhodophyta (Porphyridium cruentum) - had a strong 
434 positive effect on $T$. adhaerens growth (Fig. 6), especially in combination with the green algae 435 Chlorella sp. (Fig. 6).

436 Furthermore, the addition of Porphyridium cruentum to Chlorella sp. resulted in a 437 significant change in circularity, while feeding $T$. adhaerens with 'triple food' containing 438 Chlorella, Rhodomonas and Porphyridium showed the most pronounced effect. Culturing $T$. 439 adhaerens on either of the single foods showed similar isoperimetric values (Fig. 7).

440

\section{9-cis-RA interferes with $T$. adhaerens growth response to specific algal food} microenvironment did not show any gross morphological changes even in $10 \mathrm{nM} 9$-cis-RA during presence of 3 and $5 \mathrm{nM}$ 9-cis-RA ceased propagation and most animals developed a balloon-like phenotype, and later darkened and decomposed.

For controlled experiments, cultures fed by P. cruentum or Chlorella sp. were incubated in 450 the presence of vehicle (DMSO/ethanol) or vehicle containing 9-cis-RA at $3.3 \mathrm{nM}$ final concentration. After 24 hours of incubation in the dark, control cultures fed by P. cruentum 452 propagated normally while animals fed by $P$. cruentum and incubated with 9-cis-RA decreased 453 their area and perimeter (Fig. 8 A). At $72 \mathrm{~h}$ of incubation, all animals fed by $P$. cruentum and 454 treated by $3.3 \mathrm{nM}$ 9-cis-RA developed the balloon-like phenotype and none of them survived 90 455 hours of exposure to 9-cis-RA (Fig. 8 B and Supplementary Fig. S2). Animals transferred from 456 stationary cultures grown in a naturally established laboratory microenvironment and subsequently 
457 fed by Chlorella sp. suffered initial loses at $24 \mathrm{~h}$ of incubation despite that their appearance seemed

458 to be normal and well adopted to the new culture condition at time 0 (regarding feeding with algal

459 food and immediately prior to addition of vehicle or 9-cis-RA to the culture and $6 \mathrm{~h}$ after the

460 transfer from the parent cultures). Animals that survived the transfer and adopted to feeding by

461 Chlorella sp., were not inhibited by exposure to $3.3 \mathrm{nM} 9$-cis-RA for $24 \mathrm{~h}$ (Fig. 8 C) and even

462 showed a slight statistically not significant increase in their area and perimeter. Nevertheless, the

463 isoperimetric values of animals incubated for $24 \mathrm{~h}$ with 9-cis-RA showed a significant increase

464 indicating a decrease of growth or exhaustion of peripheral area, that is likely to contain stem cells

465 that further differentiate into the specialized cell types (Jakob et al. 2004). In contrary to animals

466 fed by P. cruentum, exposure to 9-cis-RA was not associated with the development of the balloon-

467 like phenotype and animals survived more than 250 h (Supplementary Fig. S3). In contrast to 468 control animals which started to proliferate after $100 \mathrm{~h}$, animals exposed to 9-cis-RA did not

469 proliferate between 90 and 280 h of subsequent culture (Fig. 8 D) suggesting that 9-cis-RA

470 interferes with animal response to specific food and processes necessary for animal growth and

471 propagation. The growth arrest of $T$. adhaerens caused by 9-cis-RA was reverted by addition of

472 Porphyridium cruentum indicating that a specific food constituent rather than food availability

473 interferes with 9-cis-RA regulatory potential (Supplementary Fig S4).

474

475 DISCUSSION

476 T. adhaerens is probably the closest living species to basal metazoans with only

477 four NRs

478 Trichoplax adhaerens is an especially interesting species from an evolutionary

479 perspective. It shows the most primitive metazoan planar body arrangement known with a simple 
480 dorsal-ventral polarity, the establishment of which is one of the most ancient events in evolution

481 of animal symmetries (Smith et al. 1995; Stein \& Stevens 2014). The Placozoa are disposed with

482 only a few (probably six) morphologically recognizable cell types (Jakob et al. 2004; Smith et

483 al. 2014).

484 In strong contrast to this, the T. adhaerens genome shows larger blocks of conserved

485 synteny relative to the human genome than flies or nematodes (Srivastava et al. 2008). Genome

486 analyses indicate that Placozoa are basal relative to Bilateria as well as all other diploblastic

487 phyla (Schierwater et al. 2009), but all kinds of different views are also discussed (reviewed in

488 (Schierwater et al. 2016)).

489

In concordance with this, its genome contains four (Srivastava et al. 2008) rather than 17

490 NRs, which can be found in the cnidarian Nematostella vectensis (Reitzel \& Tarrant 2009). Even

491 though it has been proposed that Placozoa lost representatives of NR6 (SF1/GCNF), TR2/TR4 of

492 the NR2 subfamily and invertebrate specific nuclear receptors (INR, clade of invertebrate-only

493 nuclear receptors with no standard nomenclature) NR1/NR4 (Bridgham et al. 2010). The reasoning

494 in this direction depends on the assumed phylogenic position of the phylum Placozoa.

495 The four NRs found in the genome of $T$. adhaerens are relatively highly related to their

496 vertebrate counterparts, RXR (NR2B), HNF4 (NR2A), COUP-TF (NR2F) and ERR (NR3B)

497 (Srivastava et al. 2008). Among them, T. adhaerens RXR and HNF4 show the highest degree of

498 identity in protein sequence and the relatedness of T. adhaerens RXR (TaRXR) to human RXR is

499 similar to that of Tripedalia cystophora RXR (jRXR) (Kostrouch et al. 1998), which has also been

500 shown to bind 9-cis-RA at nanomolar concentrations. These results suggest that TaRXR is

501 structurally and also functionally very closely related to its vertebrate counterparts, most probably

502 representing the most basal liganded NR of all Metazoa known today. 
504

\section{T. adhaerens RXR binds preferentially 9-cis-RA}

507 in T. adhaerens binds 9-cis-RA with very high affinity and shows a strong binding preference to

508 9-cis-RA over all-trans-RA similarly, as is the case in vertebrate RXRs (Allenby et al. 1993) and 509 the cnidarian TcRXR (Kostrouch et al. 1998). metabolically active enzyme, $\mathrm{L}$-malate-NADP ${ }^{+}$oxidoreductase supported by our in vivo experiments. In the search for genes that may be under the regulation of 517 TaRXR, we identified a putative orthologue of vertebrate L-malate-NADP ${ }^{+}$oxidoreductase (EC 518 1.1.1.40) and analyzed the effect of 9-cis-RA or all-trans-RA on its expression. In agreement with 519 our binding experiments, we observed induction of this enzyme's expression at low nanomolar 520 concentrations of 9-cis-RA (1 to $10 \mathrm{nM})$. Interestingly, higher concentrations of 9-cis-RA ( $30 \mathrm{nM})$

521 had a smaller effect on expression and all-trans-RA had no effect up to $30 \mathrm{nM}$ concentrations. A 522 plausible explanation for this could be that 9-cis-RA also acts as a ligand for other T. adhaerens 523 NRs which may have an opposite effect on the expression of L-malate-NADP ${ }^{+}$oxidoreductase.

524 Furthermore, 9-cis-RA could act as a supranatural ligand and the continuous occupation of TaRXR 
525 by this high affinity ligand may interfere with the normal function of the receptor within the

526 transcription initiation machinery.

527 In mammals, regulation of malic enzyme expression is mediated by a thyroid hormone

528 receptor (TR) - RXR heterodimer (Dozin et al. 1985a; Dozin et al. 1985b; Petty et al. 1989; Petty

529 et al. 1990). By showing a 9-cis-RA dependent change in the expression of the likely placozoan

530 malic enzyme orthologue in vivo, we provide indirect evidence of a conserved RXR mediated

531 regulation of gene expression. Although the expression of L-malate-NADP ${ }^{+}$oxidoreductase in

532 mammals is usually used as a factor reflecting regulation by thyroid hormone (Dozin et al. 1986),

533 it has also been shown that its cell-type associated differences depend on the expression level of

534 RXR alpha (Hillgartner et al. 1992; Fang \& Hillgartner 2000) suggesting that regulation by RXR

535 has been conserved throughout metazoan evolution while additional regulation via thyroid 536 hormone represents an innovation of Bilateria (Wu et al. 2007).

537

538 NRs form a network responding to 9-cis-RA

539 Since autoregulation and cross-regulation of NRs by their specific ligands is well 540 documented for a large number of nuclear receptors (Tata 1994), we searched if 9-cis-RA affects 541 the expression of TaRXR mRNA relative to the other T. adhaerens NRs. Our in vivo experiments

542 showed not only effects on specific gene expression in response to very low concentrations of 9543 cis-RA (at 1 or $3 \mathrm{nM}$ ), but also an additional dose-dependent reverse effect of higher 544 concentrations. This is likely to be in line with our binding experiments that suggested the 545 possibility of an additional binding site or sites with higher capacity and lower affinity. We also 546 cannot rule out that higher concentrations of 9-cis-RA affect some of the three remaining $T$. 
547 adhaerens NRs. Nevertheless, an inhibitory effect of 9-cis-RA on the expression of its cognate

548 receptor at the protein level (through protein degradation) was reported (Nomura et al. 1999).

549 Although it is not clear if 9-cis-RA is the natural ligand for RXRs (Wolf 2006; Ruhl et al.

550 2015) conserved in all metazoan phyla studied to date, we show not only that 9-cis-RA binds

551 TaRXR with nanomolar affinity but also positively regulates its expression, which resembles auto-

552 activation of several NRs in vertebrates [e.g. ER and TR (Tata 1994; Bagamasbad \& Denver

553 2011)]. Furthermore, three out of four NRs constituting the NR complement in T. adhaerens

554 respond to treatment by 9-cis-RA at transcriptional level. Two NRs, RXR itself and ERR respond

555 positively to nanomolar concentrations of 9-cis-RA, while COUP-TF, which often acts as an

556 inhibitor of specific gene expression (Tran et al. 1992), is regulated negatively by 9-cis-RA.

557 COUP-TF was recently shown to be inactivated by small hydrophobic molecules (Le Guevel et al.

558 2017). The regulatory connections of $T$. adhaerens NRs places the autoregulation and cross-

559 regulation of NRs to the base of metazoan evolution. The proposed regulatory network of $T$.

560 adhaerens NRs is schematically represented in Supplementary Fig. S5.

561

562 Food composition rather than quantity affects phenotype of $T$. adhaerens

563 At first glance, T. adhaerens seems to benefit from any source of biological material on

564 surfaces that can be digested and absorbed by its digestive system (e.g. aquarium microorganisms

565 and detritus). Feeding with certain live microorganisms in laboratory cultures, however,

566 dramatically changes the dynamics of $T$. adhaerens cultures, such as shape, size, color, body

567 transparency, growth and divisions of the animal. For example, we observed poor growth and 568 reproduction rates of $T$. adhaerens fed solely on Chlorella sp. even at a relatively high density. In 
569 contrast, cultures fed with red pigment containing Rhodomonas salina showed much faster

570 proliferation and led, in part, to the formation of giant animals, seeming to halt their division.

571 Despite Porphyridium cruentum containing similar pigments as Rhodomonas, such as

572 phycoerythrin, cultures grown with Porphyridium as the main nutrient source did not show 573 phenotypical abnormalities but the addition of it to a culture with Chlorella and Rhodomonas 574 resulted in an additive effect on reproduction rate.

575 Even though the growth of T. adhaerens seems to follow a simple program, it is likely to 576 require strict regulatory mechanisms. Formation of specific cellular types is connected with 577 phenotypic appearance of animals possessing larger proportions of certain cells, e.g. upper 578 epithelium in balloon-like animals or larger proportion of peripheral regions containing stem cell579 like cells in narrow or prolonged animals. Analysis of circularity as a measure of location specific 580 cellular proliferation is in concordance with the observed culture characteristics and shows that 581 lower isoperimetric values (less 'roundness') indicate higher reproduction rates.

582 Our experiments provide evidence that food composition is more important for $T$. 583 adhaerens growth and propagation than its quantity, which is in line with the recent finding of 584 phosphate and nitrate playing important roles determining the distribution of placozoans around 585 the globe (Paknia \& Schierwater 2015). It indicates that food constituents, especially those present 586 in the algae containing phycobilin based red pigments like Rhodomonas salina and Porphyridium 587 cruentum might possess hormone-like molecules or molecules resulting in hormone-like 588 metabolites in T. adhaerens that act through the NR complement and, indeed, analysis of NRs in 589 differently sized animals indicates impact of food composition on NR expression.

590 The high sensitivity of T. adhaerens to 9-cis-RA reflected by the transcriptional response 591 to low nanomolar concentrations of 9-cis-RA but not all-trans-RA and the interference of $3.3 \mathrm{nM}$ 
592 9-cis-RA with the animal response to feeding together with the high affinity binding of 9-cis-RA

593 by TaRXR suggests that the response of $T$. adhaerens to 9-cis-RA is mediated by TaRXR. It cannot

594 be excluded that other T. adhaerens NRs, especially TaCOUP-TF and possibly also TaHNF4 may

595 also be affected by 9-cis-RA. It seems possible that 9-cis-RA or similarly shaped molecules may

596 be present in T. adhaerens food or can be formed from retinoids and other molecular components

597 of food. Our data indicate that the sensitivity of T. adhaerens to 9-cis-RA depends on the actual

598 feeding conditions and animal growth. There are several possible scenarios that may explain the

599 high sensitivity of $T$. adhaerens to 9-cis-RA. The activation of RXR by 9-cis-RA or similar 600 compounds has been documented in vertebrates (Allenby et al. 1993; Ruhl et al. 2015; de Lera et 601 al. 2016). The observation of 9-cis-RA induced growth arrest is similar as data reported on 602 mammalian cells (e.g. (Wente et al. 2007)) however the concentration of 9-cis-RA used in our 603 experiments is approximately 30 to 3000 times lower than the levels reported in most mammalian 604 systems. It seems likely that very low concentrations of natural ligands including 9-cis-RA or 605 similarly shaped molecules or other molecules present in the algal food or produced from algal 606 food as metabolites in T. adhaerens regulate the gene expression via RXR in T. adhaerens. This 607 may be connected with $T$. adhaerens strong response to light exposure visible as coordinated 608 relocations of animals inside laboratory culture containers and a strong influence of annual seasons 609 on $T$. adhaerens propagation rates observed in laboratories localized in temperate geographical 610 zones. The 9-cis conformation of RA is not only sensitive to light exposure with its reversal to all611 trans conformation but it can also be formed by specific UV irradiation from all-trans conformation 612 up to $10 \%$ as shown by Dr. Hans Cahnmann (Cahnmann 1995).

613 Chlorophyll hydrophobic side chain which anchors the molecule to the chloroplast 614 thylakoid membrane is metabolized to phytol that was shown to act as an RXR agonist (Kitareewan 
615 et al. 1996). Other molecules called rexinoids, which often contain aromatic rings in their structure

616 act as RXR agonists or antagonists (Dawson \& Xia 2012). Lately, another group of ligands called

617 organotins was shown to affect regulation by RXR (le Maire et al. 2009). It has been proposed that

618 RXRs can bind a larger group of polyunsaturated fatty acids (docosahexaenoic acid and

619 arachidonic acid) and act as their sensors (de Urquiza et al. 2000; Lengqvist et al. 2004).

620 When viewed together, our work shows the presence of 9-cis-RA binding RXR in

621 Placozoa and argues for the existence of ligand regulated NRs at the base of metazoan evolution.

622 Our observations suggest the existence an endocrine-like regulatory network of NRs in $T$.

623 adhaerens (schematically represented in Supplementary Fig. S5). Endocrine, hormone - receptor

624 regulations involving NRs may be viewed as specialized, very powerful yet not prevailing

625 regulations transmitted by NRs. Increasingly bigger numbers of non-hormonal ligands originating

626 in environment, food or metabolism are emerging as regulatory molecules of NRs (Holzer et al.

627 2017). Our data suggest that non-hormonal, environment and food derived ligands are likely to be

628 the first or very early ligands regulating the metazoan response to food availability and

629 orchestrating growth of basal metazoans and necessary differentiation to specialized cell types. In

630 this sense, NRs in T. adhaerens represent an endocrine-like system of ancestor NRs.

631 This work suggests that ligand regulated RXR is involved in the coordination of animal

632 growth and development throughout the metazoan evolution. This also suggests that the regulation

633 by liganded NRs evolved as an evolutionary need connected with heterotrophy and 634 multicellularity.

635 In fact, despite fragments of NR domains being found in prokaryotes, no single full sized

636 NR has been discovered in bacteria or archaea and the closest known relatives to metazoans,

637 unicellular and colonial Choanoflagellates, lack nuclear receptors, as well as genes of several other 
638 regulatory pathways (King et al. 2008). On the other hand in fungi, the sister group of Holozoa,

639 (Shalchian-Tabrizi et al. 2008) transcription factors surprisingly similar to metazoan NRs evolved

640 independently possibly for the regulation of metabolism and response to xenobiotics (Thakur et

641 al. 2008; Naar \& Thakur 2009). Thus, the evolution of NRs seems to be associated with two key

642 evolutionary features of metazoans: multicellularity and heterotrophy.

643 Ctenophores, a possible sister phylum to Cnidaria, do not contain classical NRs featuring 644 both mechanistically critical domains of NRs, the DNA binding and ligand binding domains. 645 Nevertheless, the ctenophore Mnemiopsis contains two orthologues of NR2A (HNF4) that lack the 646 DNA binding domain (Reitzel et al. 2011).

647 Our observations of the exceptionally high sensitivity of $T$. adhaerens to 9-cis-RA imply 648 the possibility that the originally very strong regulations mediated by NRs might have been 649 softened or inhibited by additionally evolved mechanisms. To our knowledge, there are no reports 650 of $100 \%$ lethal effects of exposure to low nanomolar levels of 9-cis-RA in any metazoan organism. 651 It can be speculated that these mechanisms were likely to evolve to modulate 9-cis-RA's or similar 652 ligand's regulatory potential further and might involve stronger regulations by heterodimerization 653 partners of RXR and enzymatic or transport mechanisms regulating the availability of ligands in 654 cells and tissues of more recent Metazoa.

655 In conclusion, the presence of functional nuclear receptors in T. adhaerens and their 656 proposed regulatory network support the hypothesis of a basic regulatory mechanism by NRs, 657 which may have been subspecialized with the appearance of new NRs in order to cope with new 658 environmental and behavioral challenges during the course of early metazoan evolution and 659 developmental regulatory needs of increasingly more complex metazoan species. 


\section{Supplementary information}

662 Unprocessed data and additional information: Supplementary File S4 - qPCR and ddPCR data,

663 Supplementary File S5 - part A - Images of T. adhaerens incubated with specific algal food,

664 Supplementary File S5 - part B - Images of T. adhaerens incubated with specific algal food,

665 Supplementary File S6 Image analysis of T. adhaerens incubated with specific algal food,

666 Supplementary File S10 - Images of control and 9-cis-RA treated Trichoplax fed by Porphyridium

667 cruentum at time 0 and after 24 h, Supplmentary File S12 - Additional information concerning

668 the analysis of the binding of 9-cis-RA to Trichoplax adhaerens RXR. Assessment of the quality

669 of GST-RXR by polyacrylamide gel electrophoresis.

\section{Acknowledgements}

671 The databases of NCBI (Coordinators 2017) and Joint Genome Institute of United States

672 Department of Energy (http://igi.doe.gov/) (Nordberg et al. 2014) provided bioinformatics

673 support for this study.

674

\section{References}

676

677

Allenby G, Bocquel MT, Saunders M, Kazmer S, Speck J, Rosenberger M, Lovey A, Kastner P,

678

679

680

681

682

683

684

685

686

687

688

689

690 Grippo JF, Chambon P, and Levin AA. 1993. Retinoic acid receptors and retinoid X receptors: interactions with endogenous retinoic acids. Proc Natl Acad Sci US A 90:3034.

Bagamasbad P, and Denver RJ. 2011. Mechanisms and significance of nuclear receptor autoand cross-regulation. Gen Comp Endocrinol 170:3-17. 10.1016/j.ygcen.2010.03.013

Baker ME. 2008. Trichoplax, the simplest known animal, contains an estrogen-related receptor but no estrogen receptor: Implications for estrogen receptor evolution. Biochem Biophys Res Commun 375:623-627.

Bell EA, Boehnke P, Harrison TM, and Mao WL. 2015. Potentially biogenic carbon preserved in a 4.1 billion-year-old zircon. Proc Natl Acad Sci U S A 112:14518-14521. 10.1073/pnas.1517557112

Bridgham JT, Eick GN, Larroux C, Deshpande K, Harms MJ, Gauthier ME, Ortlund EA, Degnan BM, and Thornton JW. 2010. Protein evolution by molecular tinkering: diversification 
691

692

693

694

695

696

697

698

699

700

701

702

703

704

705

706

707

708

709

710

711

712

713

714

715

716

717

718

719

720

721

722

723

724

725

726

727

728

729

730

731

732

733

734

735

of the nuclear receptor superfamily from a ligand-dependent ancestor. PLoS Biol 8. 10.1371/journal.pbio.1000497

Cahnmann HJ. 1995. A fast photoisomerization method for the preparation of tritium-labeled 9-cis-retinoic acid of high specific activity. Anal Biochem 227:49-53. 10.1006/abio.1995.1251

Coordinators NR. 2017. Database Resources of the National Center for Biotechnology Information. Nucleic Acids Res 45:D12-D17. 10.1093/nar/gkw1071

Dawson MI, and Xia ZB. 2012. The retinoid X receptors and their ligands. Biochimica Et Biophysica Acta-Molecular and Cell Biology of Lipids 1821:21-56. 10.1016/j.bbalip.2011.09.014

de Lera AR, Krezel W, and Ruhl R. 2016. An Endogenous Mammalian Retinoid X Receptor Ligand, At Last! ChemMedChem 11:1027-1037. 10.1002/cmdc.201600105

de Urquiza AM, Liu S, Sjoberg M, Zetterstrom RH, Griffiths W, Sjovall J, and Perlmann T. 2000. Docosahexaenoic acid, a ligand for the retinoid $\mathrm{X}$ receptor in mouse brain. Science 290:2140-2144.

Dozin B, Cahnmann HJ, and Nikodem VM. 1985a. Identification of thyroid hormone receptors in rat liver nuclei by photoaffinity labeling with L-thyroxine and triiodo-L-thyronine. Biochemistry 24:5197-5202.

Dozin B, Magnuson MA, and Nikodem VM. 1985b. Tissue-specific regulation of two functional malic enzyme mRNAs by triiodothyronine. Biochemistry 24:5581-5586.

Dozin B, Magnuson MA, and Nikodem VM. 1986. Thyroid hormone regulation of malic enzyme synthesis. Dual tissue-specific control. J Biol Chem 261:10290-10292.

Egea PF, Klaholz BP, and Moras D. 2000. Ligand-protein interactions in nuclear receptors of hormones. FEBS letters 476:62-67.

Escriva H, Bertrand S, and Laudet V. 2004. The evolution of the nuclear receptor superfamily. Essays Biochem 40:11-26.

Escriva H, Delaunay F, and Laudet V. 2000. Ligand binding and nuclear receptor evolution. Bioessays 22:717-727. 10.1002/1521-1878(200008)22:8<717::AID-BIES5>3.0.C0;2I

Escriva H, Langlois MC, Mendonca RL, Pierce R, and Laudet V. 1998. Evolution and diversification of the nuclear receptor superfamily. Ann N Y Acad Sci 839:143-146.

Escriva H, Safi R, Hanni C, Langlois MC, Saumitou-Laprade P, Stehelin D, Capron A, Pierce R, and Laudet V. 1997. Ligand binding was acquired during evolution of nuclear receptors. Proc Natl Acad Sci U S A 94:6803-6808.

Fang X, and Hillgartner FB. 2000. Alterations in retinoid X receptor-alpha expression contribute to cell-type dependent differences in thyroid hormone regulation of malic enzyme transcription. Mol Cell Endocrinol 164:41-52.

Forman BM, and Samuels HH. 1990a. Dimerization among nuclear hormone receptors. New Biol 2:587-594.

Forman BM, and Samuels HH. 1990b. Interactions among a subfamily of nuclear hormone receptors: the regulatory zipper model. Mol Endocrinol 4:1293-1301.10.1210/mend4-9-1293

Guindon S, Dufayard JF, Lefort V, Anisimova M, Hordijk W, and Gascuel O. 2010. New algorithms and methods to estimate maximum-likelihood phylogenies: assessing the performance of PhyML 3.0. Syst Biol 59:307-321. 10.1093/sysbio/syq010 
736 Gutierrez-Mazariegos J, Schubert M, and Laudet V. 2014. Evolution of retinoic acid receptors

737

738

739

740

741

742

743

744

745

746

747

748

749

750

751

752

753

754

755

756

757

758

759

760

761

762

763

764

765

766

767

768

769

770

771

772

773

774

775

776

777

778

779

780

and retinoic acid signaling. Subcell Biochem 70:55-73. 10.1007/978-94-017-90505

Hillgartner FB, Chen W, and Goodridge AG. 1992. Overexpression of the alpha-thyroid hormone receptor in avian cell lines. Effects on expression of the malic enzyme gene are selective and cell-specific. J Biol Chem 267:12299-12306.

Holzer G, Markov GV, and Laudet V. 2017. Evolution of Nuclear Receptors and Ligand Signaling: Toward a Soft Key-Lock Model? Curr Top Dev Biol 125:1-38. 10.1016/bs.ctdb.2017.02.003

Issemann I, and Green S. 1990. Activation of a member of the steroid hormone receptor superfamily by peroxisome proliferators. Nature 347:645-650. 10.1038/347645a0

Jakob W, Sagasser S, Dellaporta S, Holland P, Kuhn K, and Schierwater B. 2004. The Trox-2 Hox/ParaHox gene of Trichoplax (Placozoa) marks an epithelial boundary. Dev Genes Evol 214:170-175.

Kana R, Kotabova E, Lukes M, Papacek S, Matonoha C, Liu LN, Prasil O, and Mullineaux CW. 2014. Phycobilisome Mobility and Its Role in the Regulation of Light Harvesting in Red Algae. Plant Physiol 165:1618-1631.10.1104/pp.114.236075

Kana R, Kotabova E, Sobotka R, and Prasil 0. 2012. Non-photochemical quenching in cryptophyte alga Rhodomonas salina is located in chlorophyll a/c antennae. PLoS One 7:e29700.

Kiefer JC. 2006. Emerging developmental model systems. Dev Dyn 235:2895-2899.

King N, Westbrook MJ, Young SL, Kuo A, Abedin M, Chapman J, Fairclough S, Hellsten U, Isogai Y, Letunic I, Marr M, Pincus D, Putnam N, Rokas A, Wright KJ, Zuzow R, Dirks W, Good M, Goodstein D, Lemons D, Li W, Lyons JB, Morris A, Nichols S, Richter DJ, Salamov A, Sequencing JG, Bork P, Lim WA, Manning G, Miller WT, McGinnis W, Shapiro H, Tjian R, Grigoriev IV, and Rokhsar D. 2008. The genome of the choanoflagellate Monosiga brevicollis and the origin of metazoans. Nature 451:783-788. 10.1038/nature06617

Kitareewan S, Burka LT, Tomer KB, Parker CE, Deterding LJ, Stevens RD, Forman BM, Mais DE, Heyman RA, McMorris T, and Weinberger C. 1996. Phytol metabolites are circulating dietary factors that activate the nuclear receptor RXR. Mol Biol Cell 7:1153-1166.

Kostrouch Z, Kostrouchova M, Love W, Jannini E, Piatigorsky J, and Rall JE. 1998. Retinoic acid X receptor in the diploblast, Tripedalia cystophora. Proc Natl Acad Sci U S A 95:13442-13447.

Kostrouch Z, Kostrouchova M, and Rall JE. 1995. Steroid/thyroid hormone receptor genes in Caenorhabditis elegans. Proc Natl Acad Sci U S A 92:156-159.

Kostrouchova M, and Kostrouch Z. 2015. Nuclear receptors in nematode development: Natural experiments made by a phylum. Biochim Biophys Acta 1849:224-237. 10.1016/j.bbagrm.2014.06.016

Kumar R, and Thompson EB. 1999. The structure of the nuclear hormone receptors. Steroids 64:310-319.

Laudet V. 1997. Evolution of the nuclear receptor superfamily: early diversification from an ancestral orphan receptor. J Mol Endocrinol 19:207-226.

Le Guevel R, Oger F, Martinez-Jimenez CP, Bizot M, Gheeraert C, Firmin F, Ploton M, Kretova M, Palierne G, Staels B, Barath P, Talianidis I, Lefebvre P, Eeckhoute J, and Salbert G. 
781

782

783

784

785

786

787

788

789

790

791

792

793

794

795

796

797

798

799

800

801

802

803

804

805

806

807

808

809

810

811

812

813

814

815

816

817

818

819

820

821

822

823

824

825

2017. Inactivation of the Nuclear Orphan Receptor COUP-TFII by Small Chemicals. ACS Chem Biol. 10.1021/acschembio.6b00593

le Maire A, Grimaldi M, Roecklin D, Dagnino S, Vivat-Hannah V, Balaguer P, and Bourguet W. 2009. Activation of RXR-PPAR heterodimers by organotin environmental endocrine disruptors. EMBO Rep 10:367-373. 10.1038/embor.2009.8

Lengqvist J, Mata De Urquiza A, Bergman AC, Willson TM, Sjovall J, Perlmann T, and Griffiths WJ. 2004. Polyunsaturated fatty acids including docosahexaenoic and arachidonic acid bind to the retinoid $\mathrm{X}$ receptor alpha ligand-binding domain. Mol Cell Proteomics 3:692-703. 10.1074/mcp.M400003-MCP200

Letunic I, Doerks T, and Bork P. 2015. SMART: recent updates, new developments and status in 2015. Nucleic Acids Res 43:D257-260. 10.1093/nar/gku949

Markov GV, and Laudet V. 2011. Origin and evolution of the ligand-binding ability of nuclear receptors. Mol Cell Endocrinol 334:21-30. 10.1016/j.mce.2010.10.017

Naar AM, and Thakur JK. 2009. Nuclear receptor-like transcription factors in fungi. Genes Dev 23:419-432. 10.1101/gad.1743009

Nomura Y, Nagaya T, Hayashi Y, Kambe F, and Seo H. 1999. 9-cis-retinoic acid decreases the level of its cognate receptor, retinoid $\mathrm{X}$ receptor, through acceleration of the turnover. Biochem Biophys Res Commun 260:729-733. 10.1006/bbrc.1999.0969

Nordberg H, Cantor M, Dusheyko S, Hua S, Poliakov A, Shabalov I, Smirnova T, Grigoriev IV, and Dubchak I. 2014. The genome portal of the Department of Energy Joint Genome Institute: 2014 updates. Nucleic Acids Res 42:D26-31. 10.1093/nar/gkt1069

Nowickyj SM, Chithalen JV, Cameron D, Tyshenko MG, Petkovich M, Wyatt GR, Jones G, and Walker VK. 2008. Locust retinoid X receptors: 9-Cis-retinoic acid in embryos from a primitive insect. Proc Natl Acad Sci U S A 105:9540-9545. 10.1073/pnas.0712132105

Paknia 0, and Schierwater B. 2015. Global Habitat Suitability and Ecological Niche Separation in the Phylum Placozoa. PLoS One 10:e0140162. 10.1371/journal.pone.0140162

Petty KJ, Desvergne B, Mitsuhashi T, and Nikodem VM. 1990. Identification of a thyroid hormone response element in the malic enzyme gene. J Biol Chem 265:7395-7400.

Petty KJ, Morioka H, Mitsuhashi T, and Nikodem VM. 1989. Thyroid hormone regulation of transcription factors involved in malic enzyme gene expression. $J$ Biol Chem 264:11483-11490.

Reitzel AM, Pang K, Ryan JF, Mullikin JC, Martindale MQ, Baxevanis AD, and Tarrant AM. 2011. Nuclear receptors from the ctenophore Mnemiopsis leidyi lack a zinc-finger DNAbinding domain: lineage-specific loss or ancestral condition in the emergence of the nuclear receptor superfamily? Evodevo 2:3. 10.1186/2041-9139-2-3

Reitzel AM, and Tarrant AM. 2009. Nuclear receptor complement of the cnidarian Nematostella vectensis: phylogenetic relationships and developmental expression patterns. BMC Evol Biol 9:230. 10.1186/1471-2148-9-230

Robinson-Rechavi M, Escriva Garcia H, and Laudet V. 2003. The nuclear receptor superfamily. J Cell Sci 116:585-586.

Ruhl R, Krzyzosiak A, Niewiadomska-Cimicka A, Rochel N, Szeles L, Vaz B, WietrzychSchindler M, Alvarez S, Szklenar M, Nagy L, de Lera AR, and Krezel W. 2015. 9-cis13,14-Dihydroretinoic Acid Is an Endogenous Retinoid Acting as RXR Ligand in Mice. PLoS Genet 11:e1005213. 10.1371/journal.pgen.1005213 
826

827

828

829

830

831

832

833

834

835

836

837

838

839

840

841

842

843

844

845

846

847

848

849

850

851

852

853

854

855

856

857

858

859

860

861

862

863

864

865

866

867

868

869

Schierwater, Holland PWH, Miller DJ, Stadler PF, Wiegmann BM, Worheide G, Wray GA, and R D. 2016. Never Ending Analysis of a Century Old Evolutionary Debate: "Unringing" the Urmetazoon Bell. Front Ecol Evol, 4:5. 10.3389/fevo.2016.00005

Schierwater B, Kolokotronis SO, Eitel M, and DeSalle R. 2009. The Diploblast-Bilateria Sister hypothesis: parallel revolution of a nervous systems may have been a simple step. Commun Integr Biol 2:403-405.

Schultz J, Milpetz F, Bork P, and Ponting CP. 1998. SMART, a simple modular architecture research tool: identification of signaling domains. Proc Natl Acad Sci U S A 95:58575864.

Shalchian-Tabrizi K, Minge MA, Espelund M, Orr R, Ruden T, Jakobsen KS, and Cavalier-Smith T. 2008. Multigene phylogeny of choanozoa and the origin of animals. PLoS One 3:e2098. 10.1371/journal.pone.0002098

Sievers F, Wilm A, Dineen D, Gibson TJ, Karplus K, Li W, Lopez R, McWilliam H, Remmert M, Soding J, Thompson JD, and Higgins DG. 2011. Fast, scalable generation of high-quality protein multiple sequence alignments using Clustal Omega. Mol Syst Biol 7:539. $10.1038 / \mathrm{msb} .2011 .75$

Smith CL, Giordano H, Schwartz M, and DeLotto R. 1995. Spatial regulation of Drosophila snake protease activity in the generation of dorsal-ventral polarity. Development 121:4127-4135.

Smith CL, Varoqueaux F, Kittelmann M, Azzam RN, Cooper B, Winters CA, Eitel M, Fasshauer D, and Reese TS. 2014. Novel cell types, neurosecretory cells, and body plan of the early-diverging metazoan Trichoplax adhaerens. Curr Biol 24:1565-1572. 10.1016/j.cub.2014.05.046

Srivastava M, Begovic E, Chapman J, Putnam NH, Hellsten U, Kawashima T, Kuo A, Mitros T, Salamov A, Carpenter ML, Signorovitch AY, Moreno MA, Kamm K, Grimwood J, Schmutz J, Shapiro H, Grigoriev IV, Buss LW, Schierwater B, Dellaporta SL, and Rokhsar DS. 2008. The Trichoplax genome and the nature of placozoans. Nature 454:955-960.

Stein DS, and Stevens LM. 2014. Maternal control of the Drosophila dorsal-ventral body axis. Wiley Interdiscip Rev Dev Biol 3:301-330. 10.1002/wdev.138

Tata JR. 1994. Autoregulation and crossregulation of nuclear receptor genes. Trends Endocrinol Metab 5:283-290.

Thakur JK, Arthanari H, Yang F, Pan SJ, Fan X, Breger J, Frueh DP, Gulshan K, Li DK, Mylonakis E, Struhl K, Moye-Rowley WS, Cormack BP, Wagner G, and Naar AM. 2008. A nuclear receptor-like pathway regulating multidrug resistance in fungi. Nature 452:604-609. 10.1038/nature06836

Tran P, Zhang XK, Salbert G, Hermann T, Lehmann JM, and Pfahl M. 1992. COUP orphan receptors are negative regulators of retinoic acid response pathways. Mol Cell Biol 12:4666-4676.

Wente W, Brenner MB, Zitzer H, Gromada J, and Efanov AM. 2007. Activation of liver X receptors and retinoid $\mathrm{X}$ receptors induces growth arrest and apoptosis in insulinsecreting cells. Endocrinology 148:1843-1849. 10.1210/en.2006-1247

Wolf G. 2006. Is 9-cis-retinoic acid the endogenous ligand for the retinoic acid-X receptor? Nutr Rev 64:532-538. 
$870 \mathrm{Wu}$ W, Niles EG, and LoVerde PT. 2007. Thyroid hormone receptor orthologues from 871 invertebrate species with emphasis on Schistosoma mansoni. BMC Evol Biol 7:150. 872

873 10.1186/1471-2148-7-150 


\section{Figure 1}

Multiple sequence alignment of selected metazoan homologues of RXR compared with TaRXR.

Aligned with ClustalO, amino acid residue types colored according to Clustal scheme in Jalview, * indicates DBD footprint residues, \# LBD footprint residues. Black box shows the DBD, red box represents the LBD. Sequences from top to bottom (organism, identifier): Trichoplax adhaerens, TaRXR ID 53515; Homo sapiens, sp|P19793|RXRA_HUMAN; Homo sapiens, sp|P28702|RXRB_HUMAN; Homo sapiens, sp|P48443|RXRG_HUMAN; Xenopus laevis, RXR alpha, sp|P51128|RXRA_XENLA; Aurelia aurita, RXR, tr|S5ZWR0|S5ZWR0_AURAU Retinoid X receptor; Tripedalia cystophora, RXR, tr|096562|096562_TRICY Retinoic acid X receptor; Drosophila melanogaster, USP, sp|P20153|USP_DROME. DNA binding domain (DBD, red line), Ligand binding domain (LBD, green line), dimerization domain (yellow line) and amino acid residues critical for 9-cis-RA binding (conserved - red rectangles, not conserved pink rectangles) are indicated. Readers with specific color preferences may download the compared sequences (Supplementary File S1) and create the Clustal scheme with different color specifications using the Jalview program (http://www.jalview.org/). 
$T a R X R$

RXRA_HUMAN

RXRB_HUMAN

RXRG HUMAN

RXRA_XENLA

S5ZWRO_AURAU

O96562_TRIC

USP_DROME

$T a R X R$

RXRA_HUMAN

RXRB_HUMAN

RXRG_HUMAN

RXRA_XENLA

S5ZWRO_AURAU

O96562_TRICY

USP_DROME

TaRXR

RXRA_HUMAN

RXRB_HUMAN

RXRG_HUMAN

RXRA XENLA

S5ZWRO_AURAU

O96562 TRICY

USP_DROME

TaRXR

RXRA_HUMAN

RXRB_HUMAN

RXRG_HUMAN

RXRA XENLA

S5ZWRO AURAU

O96562_TRICY

USP_DROME

$T a R \times R$

RXRA_HUMAN

RXRB HUMAN

RXRG HUMAN

RXRA_XENLA

S5ZWRO_AURAU

O96562_TRICY

USP_DROME

TaRXR

RXRA_HUMAN

RXRB_HUMAN

RXRG HUMAN

RXRA_XENLA

S5ZWRO_AURAU

O96562_TRICY

USP_DROME

$T a R \times R$

RXRA_HUMAN

RXRB_HUMAN

RXRG_HUMAN

RXRA_XENLA

S5ZWRO_AURAU

O96562_TRICY

USP_DROME

TaRXR

RXRA_HUMAN

RXRB HUMAN

RXRG_HUMAN

RXRA_XENLA

S5ZWRO_AURAU

O96562_TRICY

USP_DROME

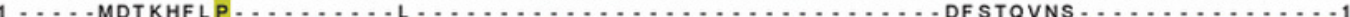

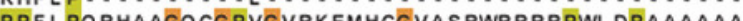
....... LGGRT . . . . CADTLRCTTSWTAGY

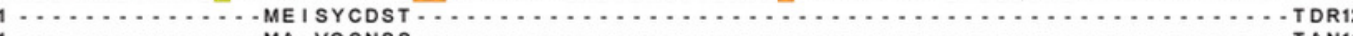

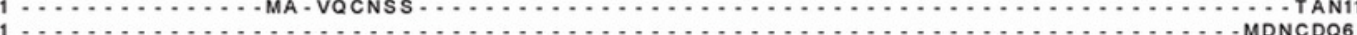

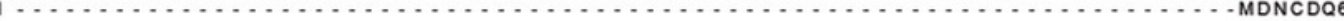

$18 \ldots .$. S - LTSPTGRGS ... MAAPSLHPSLGP . . . . . . . GIGSPGQLHSPISTLSSPINGMGPPFSVISSPMGPH75 79 DSRSPDSSSPNPLPQGVPPPSPPGPPLPPSTAPSLG . . . . . . GSGAPP . . . . PPPMPPPPLGSPFPVISSSMGSP143 $19 . . . . . .$. PGHTGS ...... TSMSPSAAL STGKPMDSHPSYTDTPVSAPRTLSAVGTPL NALGSPYRVITSAMGPP80 42 .... SSLSSSGLRGS ... MTAPL HPSLGN

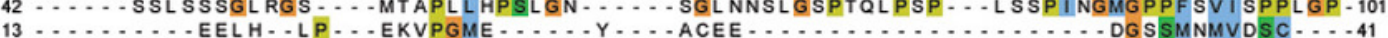

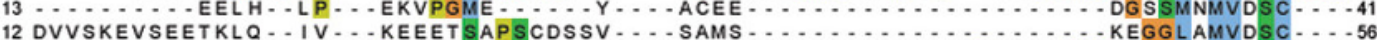
7 DASFRLSHIKEEVK ..... PDISQLNDSNNSS .................

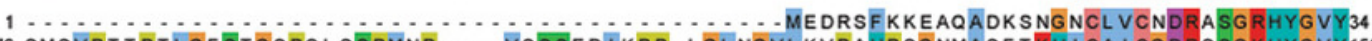
76 SMSVPT TPTLGFSTGSPQL SSPMNP. . . . VSSSEDIKPP-LGLNGVLKVPAHPSGNMASFTKHICA I CGDRSSGKHYGVY150 144 GLPPPAPPGFSGPVSSPQ INSTVSLPGGGSGPPEDVKPPVLGVRGL... - HCPPPPGGPGAGKRLCA ICGDRSSGKHYGVY220 81 SGAL AAPPG I NL VAPPSSQL NVVNS -...VSSSED I KPL - PGLPG I GNMN - YPSTSPGSLVKHICAICGDRSSGKHYGVY154 102 SMA I PSTPGLGYGTGSPQ I HSPMNS - ..-VSSTEDI KPP-PG I NG I L KVPMHPSGAMASFT KH I CA I CGDRSSGKHYGVY176 $42 \ldots$... LEPPPLESI . . . DSYSPLSGSDGTPGSSSS ..... SL SFFPQNSSPNSNGSERQMLPCAVCSDKAYVKHYGVV107 $57 \ldots$... KEASPLESI ... HPYSPLASDASGSSTSPI ....... ASSSLLQ - LPSLTADSQRPVQPCSVCSDKAYVKHYGVF 121 $55 \ldots . .$. PGSNSA ....... SSNNNSAGDAQ - MAQAPNSAGGSAAAAVQQQYPPNHPLSGSKHLCSICGDRASGKHYGVY119 DBD

35 SCEGCKGFFKRTVRKNLTYTCRDNRNCDIDKKQRNRCQYCRYQKCLQVGMKQEAVQEERVKNSTPTSKTTLPIAIAD - - - 111 151 SCEGCKGFFKRTVRKDLTYTCRDNKDCL I DKRQRNRCQYCRYQKCLAMGMKREAVQEERQRGKDRNENE - . . . . . . 219 221 SCEGCKGFFKRT I RKDLTYSCRDNKDCTVDKRQRNRCQYCRYQKCLATGMKREAVQEERQRGKDKDG-D...........288 155 SCEGCKGFFKRT I RKDL I YTCRDNKDCL I DKRQRNRCQYCRYQKCL VMGMKREAVQEERQRSRERAESE - . . . . . . 223 177 SCEGCKGFF KRT VRKDL TYTCRDSK DCM I DKRQRNR CQYCRYQ KCL AMGMKREAVQEERQRGKERNENE -..........-245 108 ACEGCKGFF KRSVRNNRKYQCLGNQRCDIDRKSRNK CQYCRFQKC IEVGMKPEAVQDETLKKEKRESTKRKAANPVSSGS187

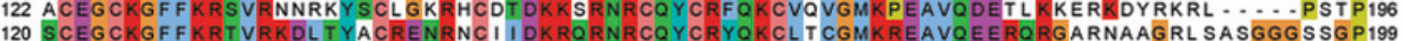

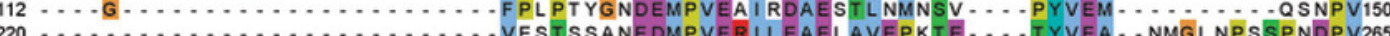
224 . 188 K - GSP - N $188 \mathrm{~K} \cdots$. . GSP . . . . . . . . . . . VEVT SSRVEMPLVP I DLVVSAESMVEPS IQ . . . LFA . . . . . . NTAVDPI231

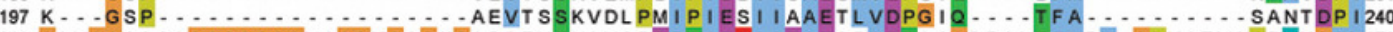
200 GSVGGSSSQGGGGGGGVSGGMGSGNGSDDFMTNSVSRDFSI ER I IEAEQRAETQCGDRALTFLRV-GPYSTVQPDYKGAV278

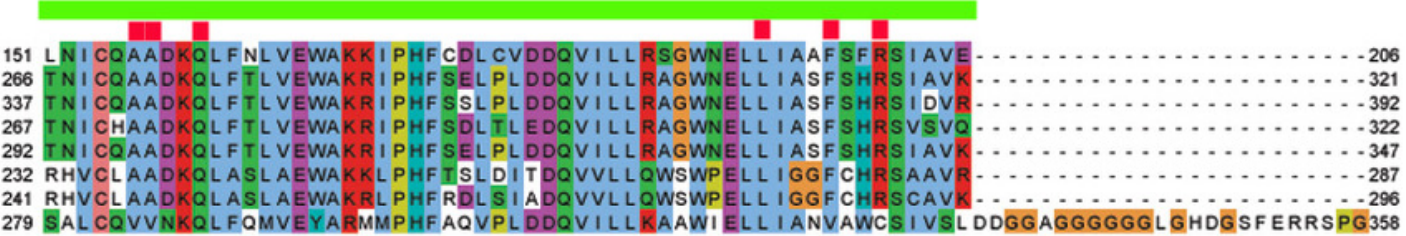

LBD

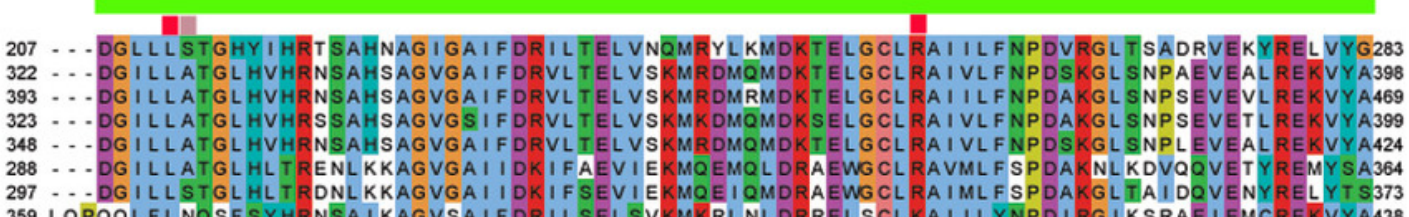

Dimerization

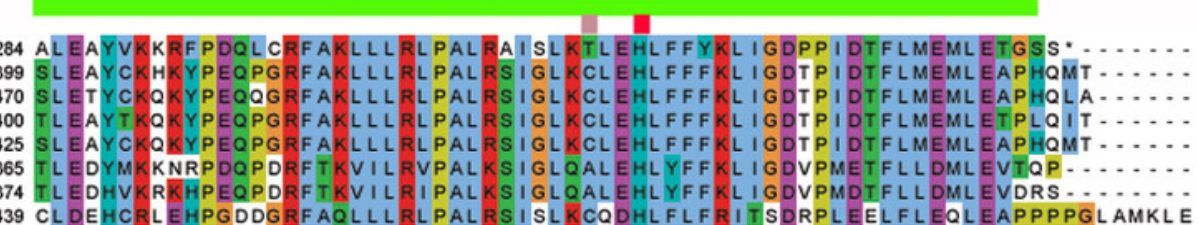




\section{Figure 2}

Binding of retinoic acids to TaRXR.

A - Single point analysis of binding preference of $T$. adhaerens RXR (thrombin cleaved) to ${ }^{3} \mathrm{H}$ labelled 9-cis-RA over all-trans-RA. Radioactive 9-cis-RA (9-cis-RA*) binds at a concentration of $4 \mathrm{nM}$ to 200 nanograms of $T$. adhaerens RXR. 200 -fold excess of unlabeled 9-cis-RA displaces more than $80 \%$ of labeled 9-cis-RA from binding to $T$. adhaerens RXR (9-cis-RA* + 9-cis-RA) while the same molar excess of all-trans-RA (9-cis-RA* + AT-RA) which is likely to contain approximately $1 \%$ spontaneously isomerized 9-cis- RA, competes away less than 50 $\%$ of bound ${ }^{3} \mathrm{H}$-labeled 9 -cis-RA. Radioactive ${ }^{3} \mathrm{H}$-labeld all-trans-RA (AT-RA ${ }^{*}$ ) at identical conditions binds only slightly more than the observed non-specific binding. This interaction is not displaced by the excess of non-labeled 9-cis-RA (AT-RA* + 9-cis-RA) nor non-labeled all-trans-RA (AT-RA* + AT-RA). Results are expressed as a ratio of the radioactivity bound to TaRXR / total radioactivity used for the binding at the given condition. B - Analysis of binding properties of $T$. adhaerens RXR (in the form of GST-TaRXR) to ${ }^{3} \mathrm{H}$-labelled 9-cis-RA and ${ }^{3} \mathrm{H}$ labelled all-trans-RA. The experiment differs from the experiment shown in A in 5-fold greater amount of radioactive all-trans-RA (and therefore only 40 -fold excess of non-radioactive competitors). The experiment shows identical binding properties of GST-TaRXR as those observed with thrombin cleaved TaRXR. C - Kinetic analysis of binding of ${ }^{3} \mathrm{H}$-labeled 9-cis-RA to $T$. adhaerens RXR prepared as GST-fusion protein (GST-TaRXR). The plateau is reached at around 3 to $5 \times 10^{-9} \mathrm{M}$. 
A

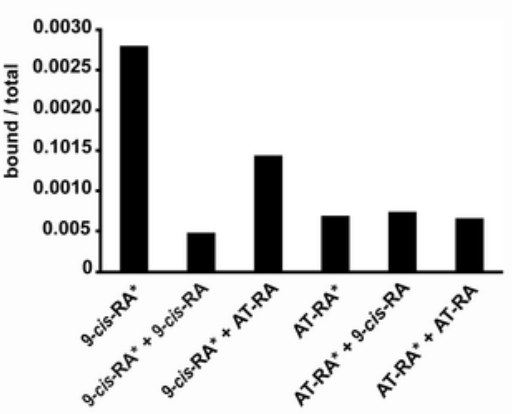

B

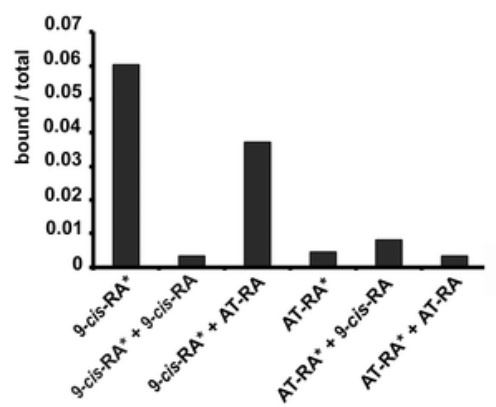

C

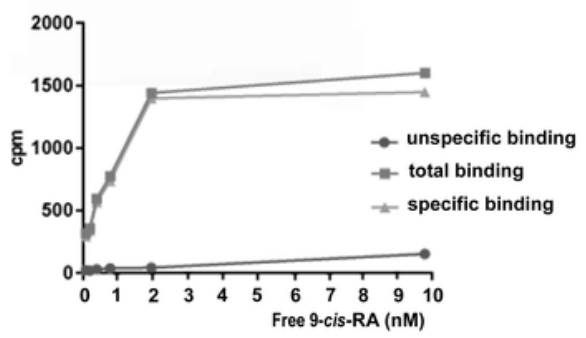




\section{Figure 3}

The effect of 9-cis-RA on the expression of the $T$. adhaerens closest putative homologue and likely orthologue of L-malate-NADP ${ }^{+}$oxidoreductase (EC1.1.1.40).

Ten to fifteen animals were cultured in the dark overnight with indicated ligands or in medium containing only the solvent used for ligand solutions. Total RNA and CDNA were prepared using identical conditions and diluted to the same working concentration suitable for ddPCR. In repeated experiments, incubation with $3 \mathrm{nM}$ 9-cis-RA induced expression of the putative $T$. adhaerens L-malate-NADP+ oxidoreductase more than 4 times. Incubation with 30 nM 9-cis-RA induced enzyme expression also, but to a lesser extent and $30 \mathrm{nM}$ all-trans-RA (AT-RA) did not upregulate the expression of the predicted L-malate-NADP ${ }^{+}$oxidoreductase.

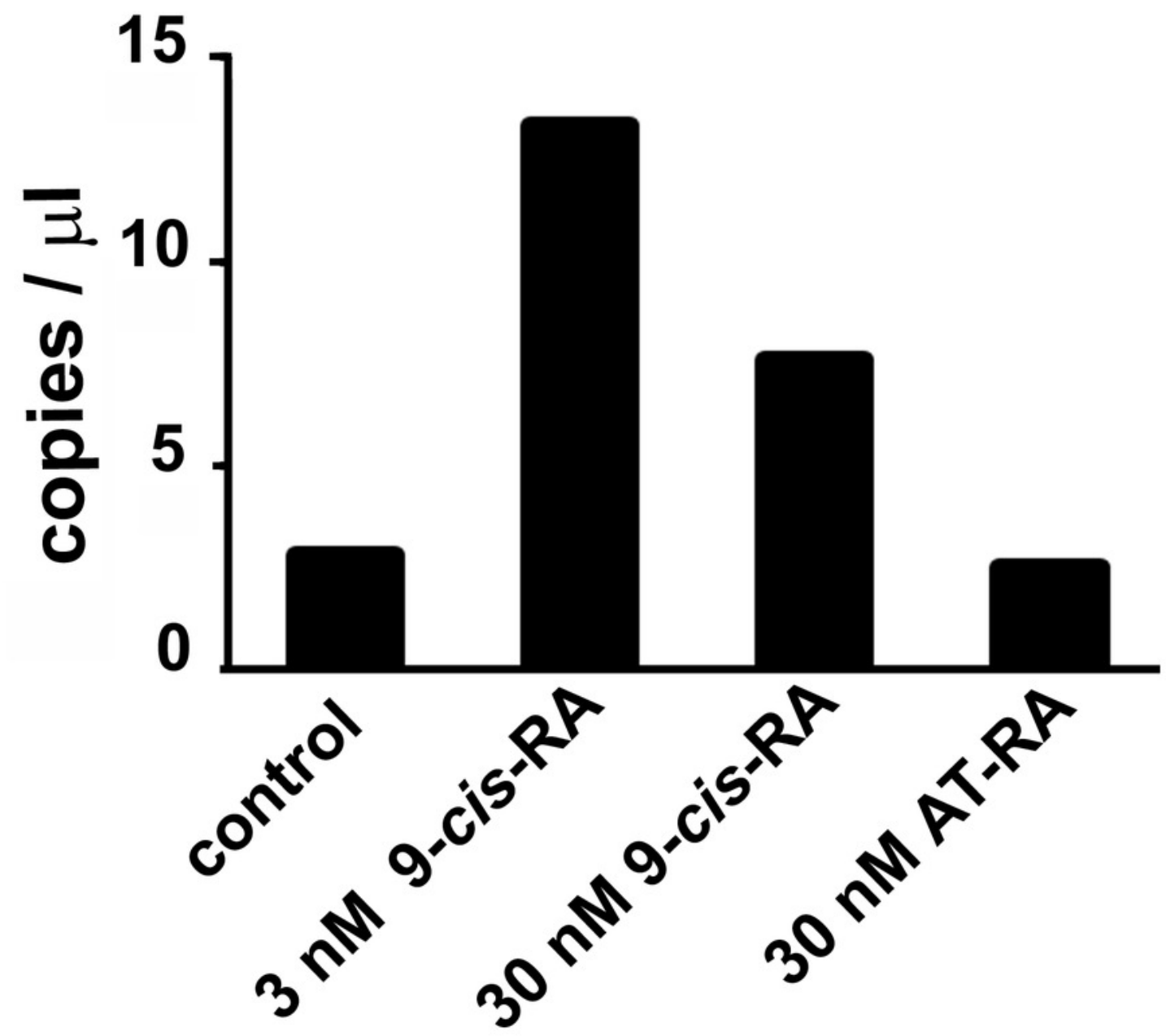




\section{Figure 4}

The effect of 9-cis-RA on the expression of $T$ adhaerens NRs.

A representative experiment of the expression of $T$. adhaerens NRs in animals exposed to various concentrations of 9-cis-RA expressed as a ratio of obtained values compared to the control using ddPCR. One and 3 nM 9-cis-RA upregulate RXR and ERR, but downregulate COUP-TF. The expression of $T$. adhaerens HNF4 is not affected by 9-cis-RA. The effect of the exposure to 9-cis-RA is stronger in the case of $1 \mathrm{nM}$ 9-cis-RA compared to $3 \mathrm{nM}$ 9-cis-RA. The exposure to $30 \mathrm{nM}$, as well as $50 \mathrm{nM}$ concentrations of 9-cis-RA reverse the effect of 9-cis-RA on the expression of RXR and COUP-TF, but do not influence the expression of ERR. The level of expression of HNF4 is not changed by exposure of $T$. adhaerens to various concentrations of 9-cis-RA. The data suggest that a network sensitive to nanomolar concentrations of 9-cisRA at the expressional level is formed by RXR, COUP-TF and ERR. All four NRs have conserved P-box (regions responsible for binding to response elements (RE) in gene promoters) and are likely to bind overlapping REs and to form a functional network.

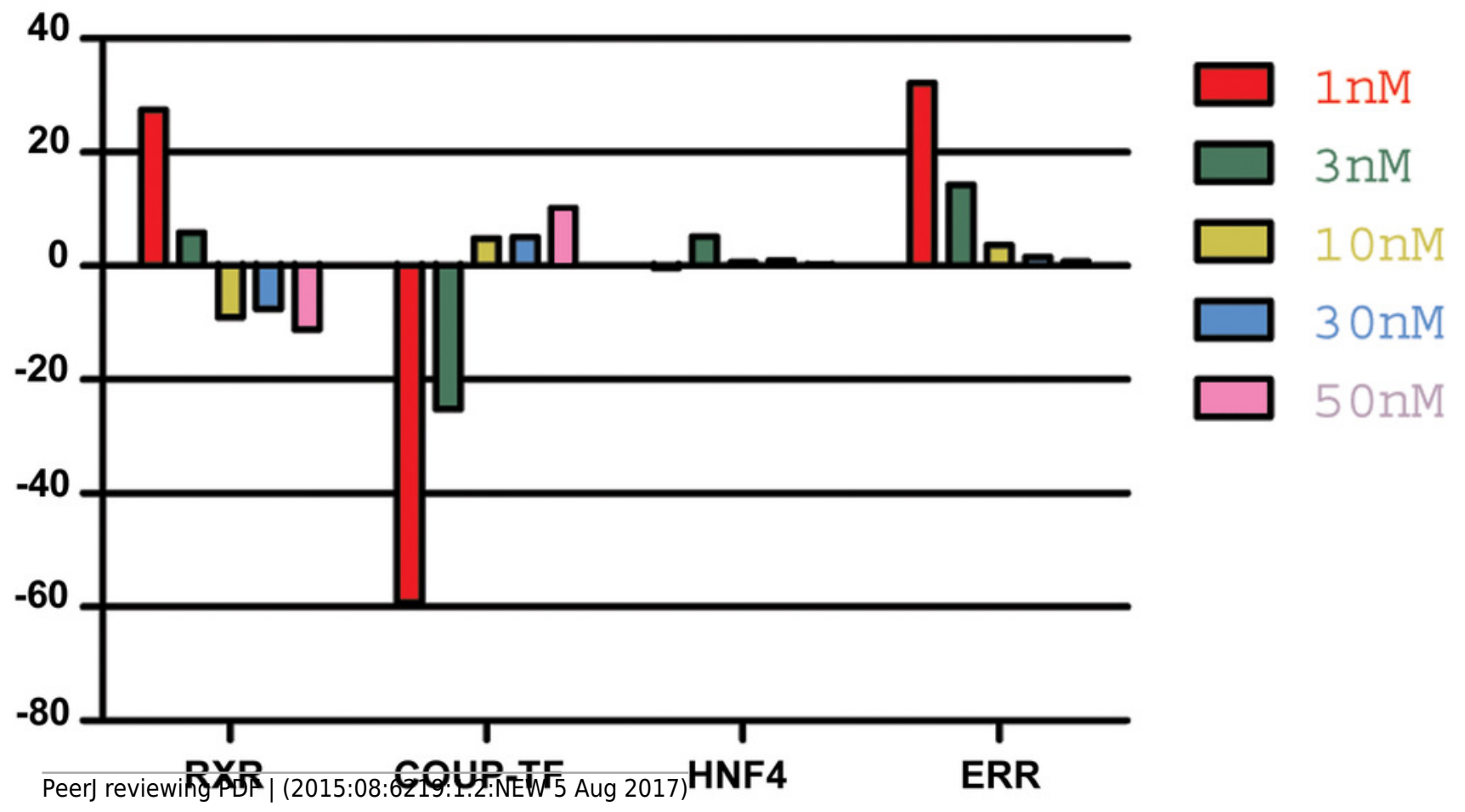




\section{Figure 5}

Phenotypes of $T$. adhaerens change at various feeding conditions.

T. adhaerens acquires various body shapes in individual cultures dependent on food availability and composition. At conditions maintained in stable and biologically equilibrated stock aquariums, $T$. adhaerens is usually small and pale with diameter varying from $50 \mu \mathrm{m}$ to $400 \mu \mathrm{m}$ while cultures with added algae contain large flat animals with diameter reaching up to $1 \mathrm{~mm}$ ( $A$ and $B)$. In some cultures, animals grow as long stretching structures, reaching a length exceeding one or even several centimeters (C). The algal food makes the animals greenish, reddish, rusty or brown with variable proportion of prominent dark cells. Animal shapes also vary from flat and round with smooth circumference, to curved or ruffled circumference or animals with long projections. Bars represent $1 \mathrm{~mm}$ in $A, B, I, 1 \mathrm{~cm}$ in C, 250 $\mu \mathrm{m}$ in $\mathrm{D}, 500 \mu \mathrm{m}$ in $\mathrm{E}$ and $\mathrm{H}, 200 \mu \mathrm{m}$ in $\mathrm{F}$, and $100 \mu \mathrm{m}$ in $\mathrm{G}$.

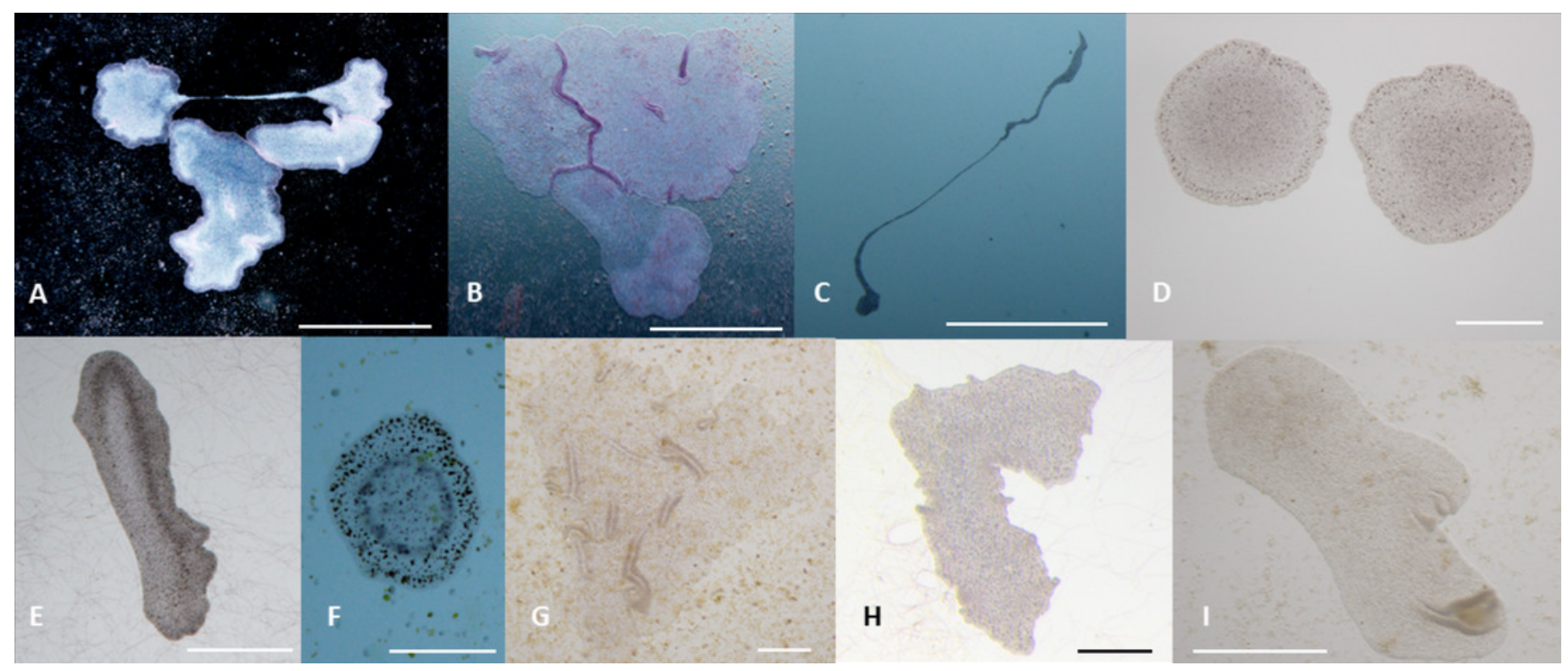


Figure $\mathbf{6}$ (on next page)

Propagation of $T$. adhaerens depends on algal food composition.

Three cultures of 5 large animals in each were established and fed with the same number of algal cells consisting of Chlorella sp., Chlorella sp. and Rhodomonas salina and Chlorella sp., Rhodomonas salina and Porphyridium cruentum. While the culture fed with Chlorella sp. only doubled in the number of animals within a period of one week, cultures with red pigment containing algae multiplied more than 5 times and 10 times within the same time period. 


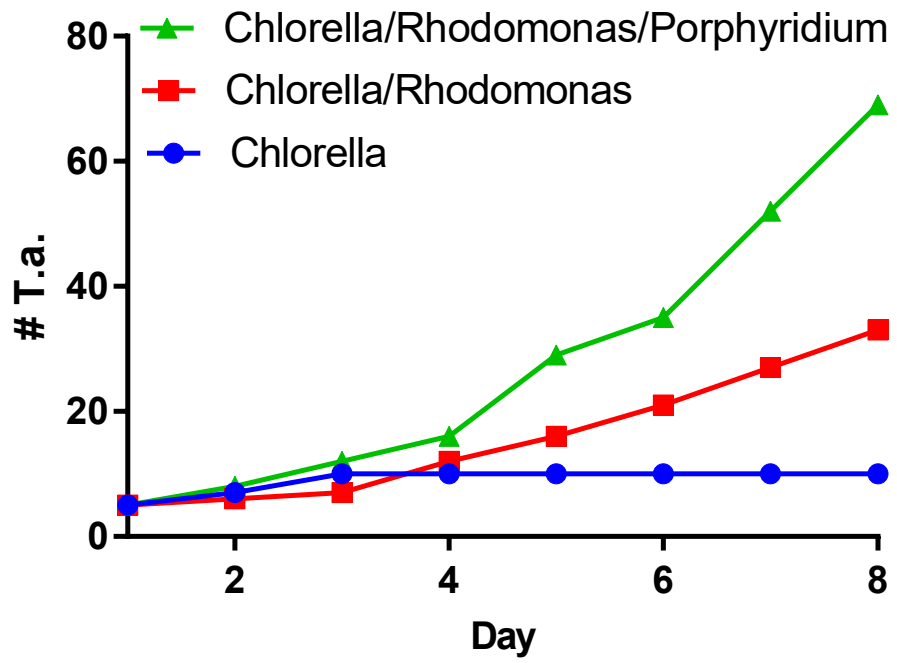




\section{Figure 7}

The effect of algal food composition on $T$. adhaerens growth and appearance.

T. adhaerens was cultured similarly as shown in Fig. $\mathbf{6}$ and all animals were photographed and analyzed using ImageJ program for their number (panel A), mean area (panel B), mean perimeter (panel C), mean perimeter/area ratio (panel D) and mean isometric quotient (panel E) after one week. Ch - stands for feeding with Chlorella sp., Rh - Rhodomonas salina, Po Porphyridium cruentum, and their combinations. 3Rh stands for a culture with three times higher concentration of Rhodomonas salina and $3 \mathrm{Ch}$ for three times higher concentration of Chlorella sp. Panel A shows that addition of Rhodomonas salina $(\mathrm{Ch}+\mathrm{Rh})$ greatly increases the number of animals observed after one week of culture. This effect is even more pronounced in cultures containing all three algae, while three times bigger concentration of only one type of algae ( $\mathrm{Ch}$ and $\mathrm{Rh}$ ) has little or no effect. This is even more pronounced when the area and perimeter are determined (panels $\mathrm{B}$ and $\mathrm{C}$ ). Determination of the isoperimetric quotient in individual cultures indicates that cultures with Rhodomonas salina have a significantly smaller ratio, suggesting higher proliferative rate of structures at the animal circumference (panel E). Bars represent 95\% confidence interval. Raw data are provided as Supplementary File S5 and S6. 
A

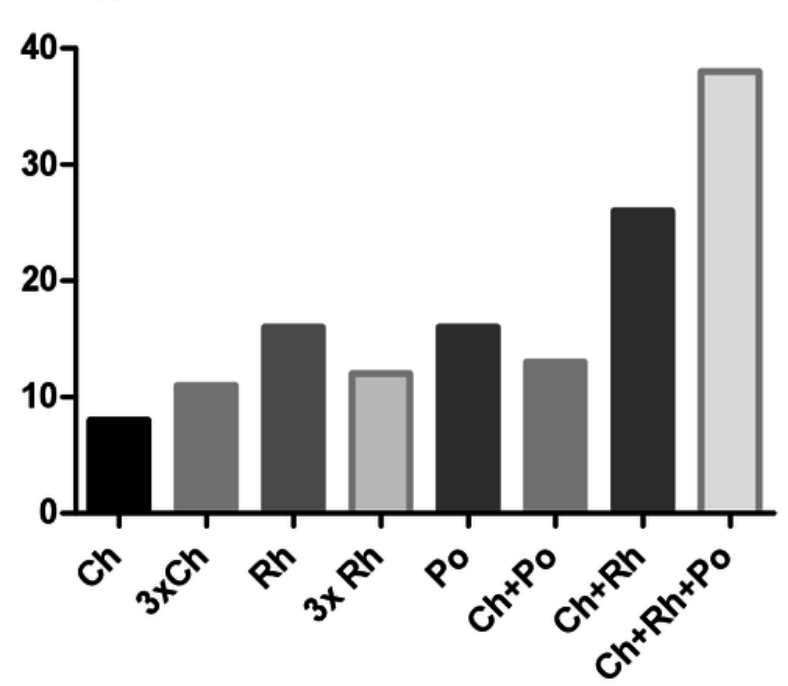

C
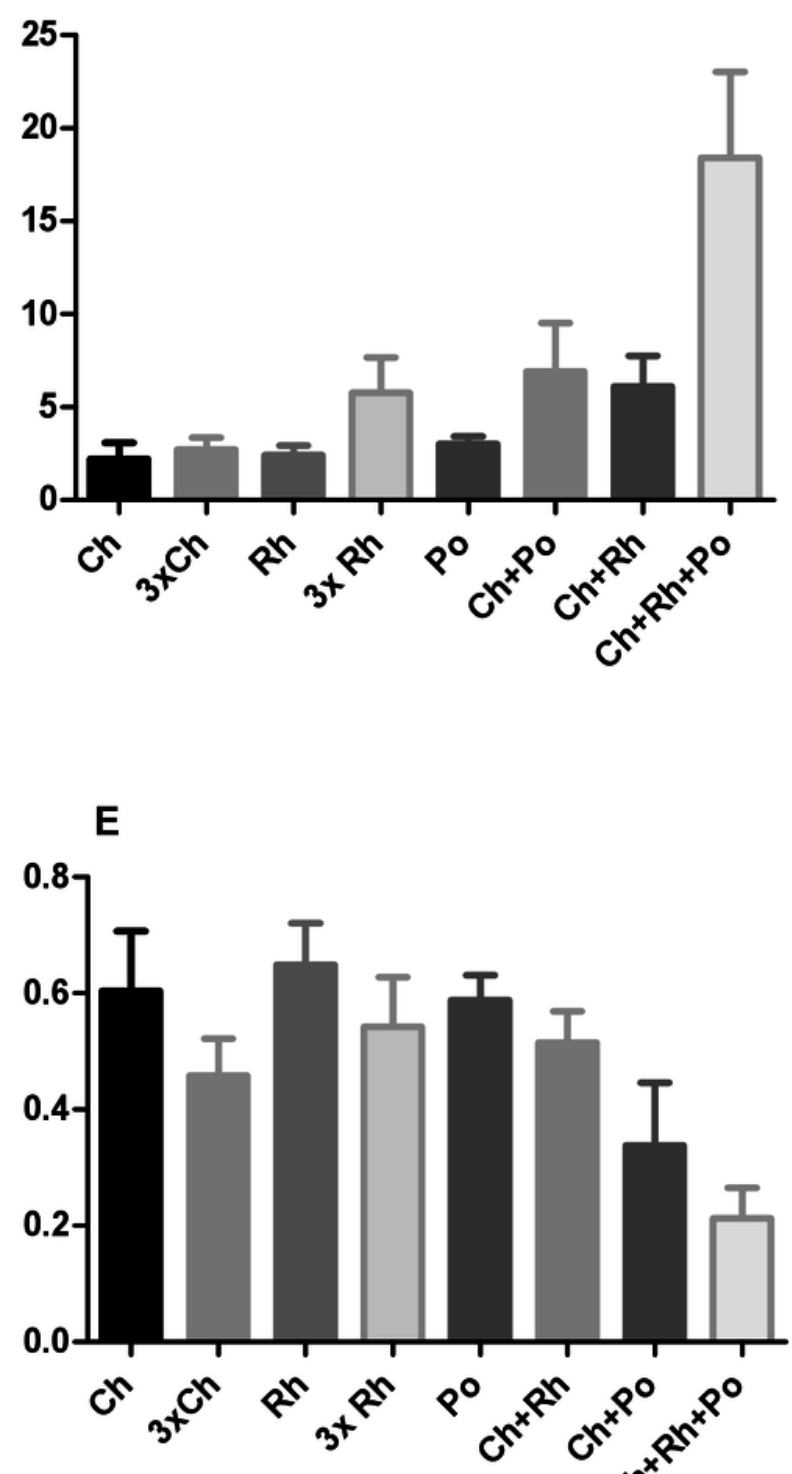
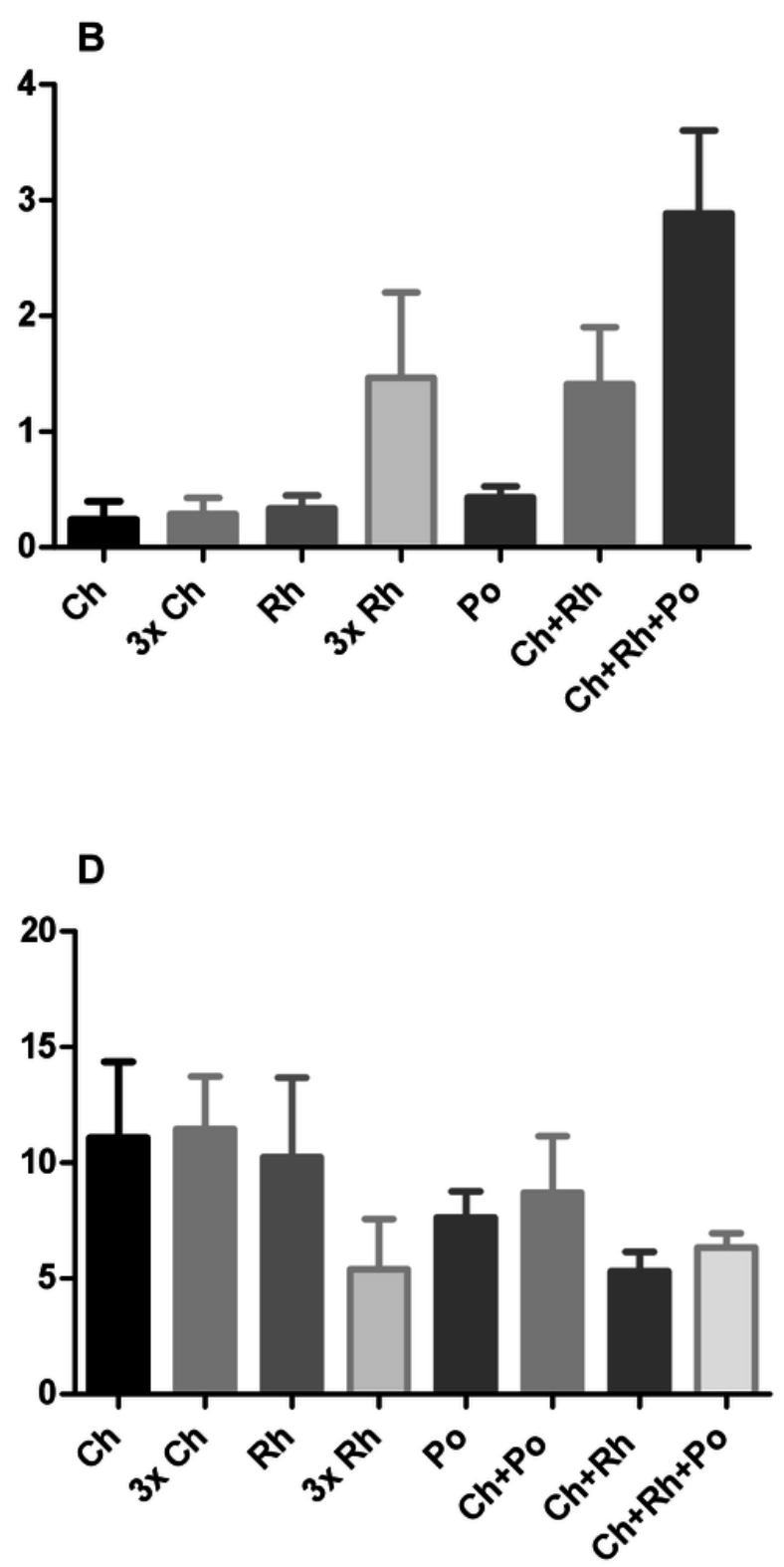


\section{Figure 8}

The effect of 9-cis-RA on growth of $T$. adhaerens fed by Porphyridium cruentum or Chlorella sp.

Panel A shows the comparison of the total area and total perimeter of control $T$. adhaerens and $T$. adhaerens treated by 9 -cis-RA for $24 \mathrm{~h}$ expressed as arbitrary units derived from pixel measurements obtained at $24 \mathrm{~h}$ and compared to values obtained immediately prior to incubation. The data indicates that animals incubated in $3.3 \mathrm{nM}$ 9-cis-RA decreased their area and perimeter to approximately $50 \%$ in comparison to control animals. Panel B shows the development of cultures over a three-week period. The animals treated by 9-cis-RA developed a balloon-like phenotype at $72 \mathrm{~h}$ of incubation and died at $90 \mathrm{~h}$ of incubation. Panels $C$ and $D$ show the data obtained with $T$. adhaerens originating from cultures fed by naturally established biofilms in laboraotory aquariums, fed by Chlorella sp. and treated similarly as shown in panel A and B. Panel $E$ shows the analysis of circularity of animals presented in panel $\mathrm{A}$ and documents that animals treated by 9-cis-RA increased their circularity already at $24 \mathrm{~h}$ of exposure suggesting arrest of growth of animal peripheral tissues. 


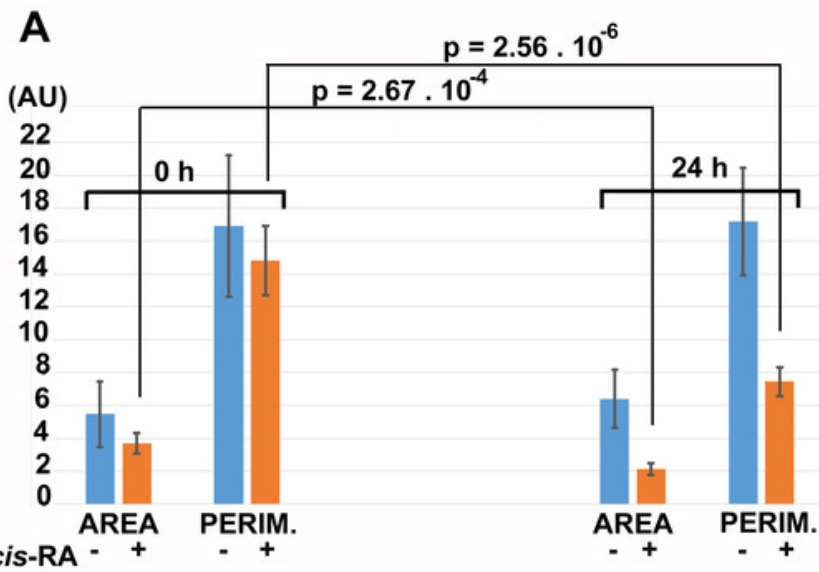

B

(No of animals)

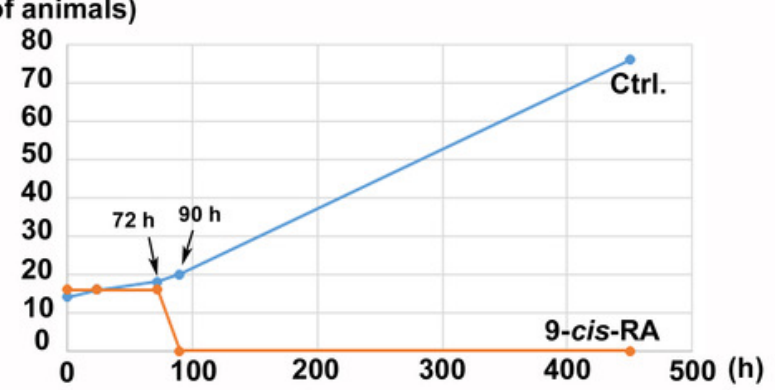

9-cis-RA -

NS

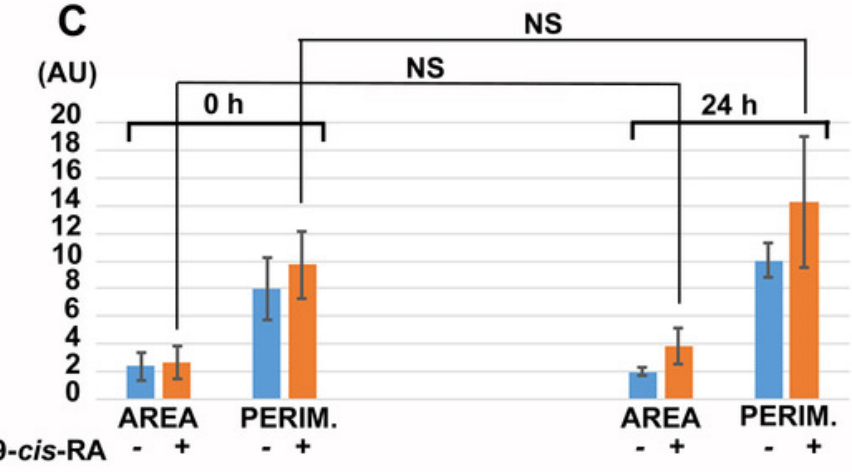

D

(No of animals)

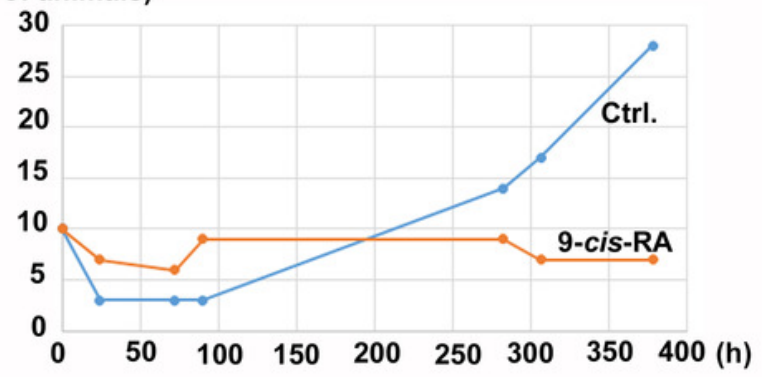

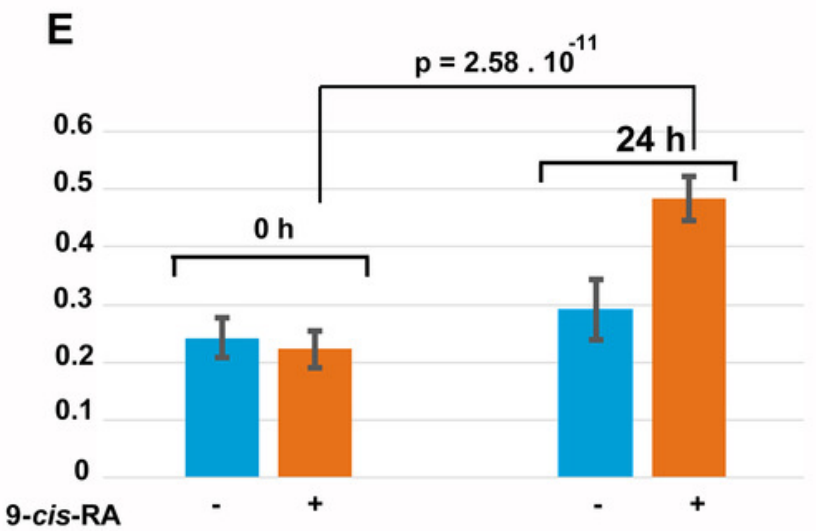

